\title{
Dynamical Modularity of the Genotype-Phenotype Map
}

Johannes Jaeger ${ }^{1} \&$ Nick Monk ${ }^{2}$

1. Complexity Science Hub (CSH) Vienna, Austria

2. School of Mathematics and Statistics, University of Sheffield, Sheffield, UK

\begin{abstract}
An organism's phenotype can be thought of as consisting of a set of discrete traits, able to evolve relatively independently of each other. This implies that the developmental processes generating these traits - the underlying genotype-phenotype map-must also be functionally organised in a modular manner. The genotype-phenotype map lies at the heart of evolutionary systems biology. Recently, it has become popular to define developmental modules in terms of the structure of gene regulatory networks. This approach is inherently limited: gene networks often do not have structural modularity. More generally, the connection between structure and function is quite loose. In this chapter, we discuss an alternative approach based on the concept of dynamical modularity, which overcomes many of the limitations of structural modules. A dynamical module consists of the activities of a set of genes and their interactions that generate a specific dynamic behaviour. These modules can be identified and characterised by phase-space analysis of data-driven models. We showcase the power and the promise of this new approach using several case studies. Dynamical modularity forms an important component of a general theory of the evolution of regulatory systems and the genotype-phenotype map they define.
\end{abstract}

\section{Introduction: modular traits and the genotype-phenotype map}

The question why organisms are able to evolve is one of the most fundamental and unresolved questions in biology today. What kind of systems architecture permits and facilitates the generation of adaptive variation? What are the indispensable characteristics of such an architecture? Modularity is one of the main candidates for such a fundamental structural property of evolving systems. George Cuvier already argued that the functional integration of parts implies that evolution cannot occur; but since then, we have discovered modular elements at all levels of living systems (Schlosser \& Wagner, 2004; Callebaut \& RasskinGutman, 2005). At the molecular level, genes and their regulatory sequences are thought to be organised in a modular way. At the phenotypic level, the study of evolution relies on the subdivision of the organism into individual characters or traits. These traits provide the foundation for establishing character homologies and their phylogenetic relationships (Wagner 2014).

Lewontin $(1970,1974)$ emphasises the "quasi-independence" of evolutionary characters: they 
can be individuated through functional or structural criteria (see, for example, Riedl 1975), or in terms of their variational properties (Wagner \& Alternberg 1996). In this sense, traits are functional, structural, and/or variational modules (Fig. 1A). Without modularity limiting pleiotropic effects, selection for specific characters would be impossible, and coherent description of evolutionary processes would be unattainable. It may be that some features of an organism may not be separable in this way ${ }^{1}$, but evolutionary theory at a minimum focuses attention on the set of features that do exhibit a significant degree of quasi-independence. This is why it is widely accepted that modularity is a prerequisite for evolvability, the capacity to produce adaptive change (Dawkins 1989, Wagner \& Altenberg 1996, Pigliucci 2008).

Herbert Simon $(1962,1973)$ argues further that "near-decomposability" is a necessary property of complex adaptive systems that evolve under selective pressure. It is characterised by the scarcity of strong interactions between systems parts. Near-decomposability provides a powerful principle for plausible simplification - and hence understanding - of complex adaptive systems by subdividing our explanatory tasks into manageable chunks (Callebaut 2005).

Character traits-considered as individuated features of an organism's phenotype-are generated by metabolic, physiological, and developmental processes, which constitute a mapping from genotype to phenotype (Fig. 1B) (Burns 1970, Alberch 1991, Pigliucci 2010) ${ }^{2}$. These processes compose the epigenotype of the organism (Waddington 1942, 1953, Goodwin 1982, Oster \& Alberch 1982). Evolutionary change in a phenotypic trait requires corresponding changes in its generative processes (Waddington 1957, Wagner 1989, Amundson 2005, Wagner 2014). Since phenotypic variability reflects variability in these processes, the genotype-phenotype map must have a modular architecture as well. Needham (1933) coined the term "dissociability" to describe that generative processes or subsystems are to some degree separable from each other. This suggests that variation in a given individuated trait can be mapped to specific modular subsystems of the epigenotype (Fig. 1). In this chapter, we ask how this is best achieved ${ }^{3}$. After all, understanding the modular architecture of the genotype-phenotype map is the key to reconnecting phylogeny and ontogeny, a central aim of evolutionary developmental biology (Needham 1933, Waddington

1. Gould and Lewontin (1979) use the human chin as an example to criticise the concept of character traits as natural kinds. The chin is not individuated, since it develops from the interaction of two different generative processes (the alveolar and mandibular growth fields), and thus cannot vary independently from other features of the human skull.

2. Actually, a more accurate view is to consider the central mapping to occur from phenotype to phenotype over the life cycle of an organism. On this view, the genotype provides the parameters of this dynamic map (rather than a source range as shown in Fig. 1B). Genotype space becomes embedded in phenotype space. Here, we use the metaphor of the genotype-phenotype map as a useful idealisation. It should not be interpreted in the sense that genotype space is physically or logically independent of the phenotype space. Rather, it highlights the fact that we should focus more attention on the processes that connect genotype and phenotype. Metaphors are only useful as conceptual tools when applied within their proper limitations.

3. Another interesting question is how the modular structure of the epigenotype evolved in the first place. This is an important and active field of investigation (reviewed in Wagner 1996, Wagner et al. 2007; see also Wagner 2014). We will not pursue this question any further here. 
1957, Goodwin 1982, Oster \& Alberch 1982, von Dassow \& Munro 1999, Bolker 2000, Wagner et al. 2007, 2014). Unfortunately, the nature of modularity remains elusive.

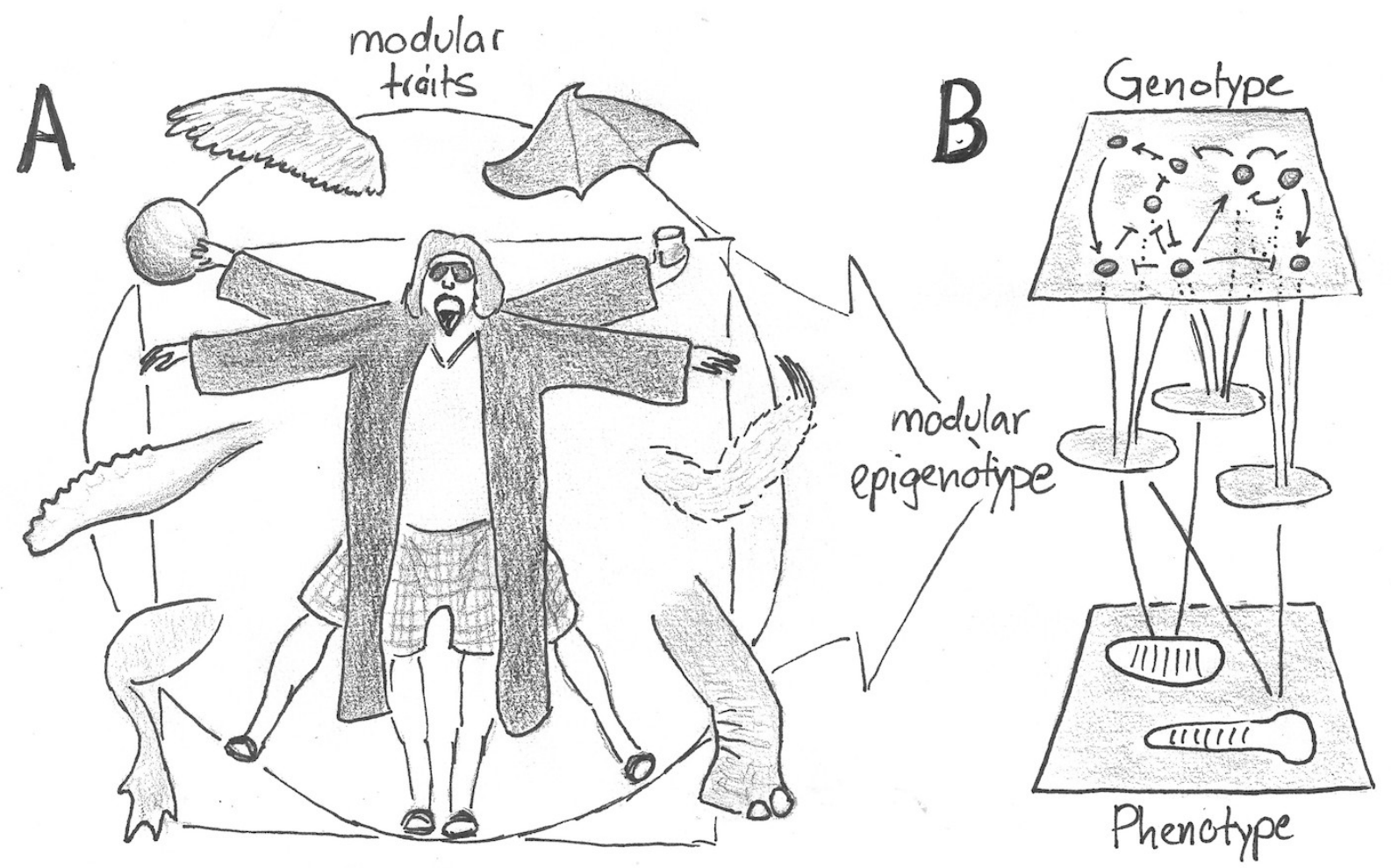

Figure 1: (A) Character traits are functional, structural and/or variational modules, each of which can undergo adaptation quasi-independently of others. (B) This implies that the genotype-phenotype map, consisting of the generative processes that produce traits, must also be structured in a modular manner. The argument in this chapter shows that such modularity must occur at the level of the epigenotype, which provides the bridge between genotype and phenotype space. With apologies to the Coen Brothers, Leonardo Da Vinci, and Günter Wagner.

The first difficulty is to precisely define what a module actually is. A general definition should apply across a broad range of specific cases, types of modules, and levels of the organisational hierarchy (Bolker 2000). In its broadest sense, a module is "a unit that is a component part of a larger system and yet possessed of its own structural and/or functional identity" (Moss 2001, p. 91). The unit could be any biological entity (a structure, a process, or a pathway) or collective of entities with more internal than external cohesion (Raff 1996, Hartwell et al. 1999, von Dassow \& Munro 1999, Bolker 2000, Callebaut 2005, DiFrisco, 2018; see also Collier 1988, 2004). In this sense, modules can be considered biological individuals (Hull 1980). They can be delineated from their surroundings and their behaviour and function emerges as a result of the integrated activity of their parts. Modules require both tight internal cohesion (their 'cohesion regime'; Collier 1988, 2004, DiFrisco, 2018) and relative independence from other modules (cf. Simon 1962, 1973). They can be defined in terms of their reusability in different contexts (Mireles and Conrad, 2018). Importantly, in the context of our argument, modules must be extended in time: they must persist for long enough to exert their effect (Callebaut 
2005). Finally, as the terms "near-decomposability" and "quasi-independence" imply, modularity is a matter of degree (Wagner \& Altenberg 1996). All modules interact with their context to some extent, usually in a structured and hierarchical way (Needham 1933, Riedl 1975, Raff 1996, Bolker 2000).

There are many different ways in which we can distinguish different types of modules. Characters can quite obviously be considered morphological modules (Lewontin 1970, 1974), and we have already encountered variational modules as a way to individuate traits (Wagner \& Alternberg 1996, Melo et al. 2016). Variational modules are often also functional modules, as shared function implies an increased tendency to co-vary (Wagner et al. 2007). Moreover, they are closely related to the concept of an evolutionary module, which informs the notion of homology and the unit-of-selection debate (e.g. Lewontin 1970, Raff 1996, Wagner 1996, Brandon 1999, Callebaut 2005, Wagner 2014). Variational/evolutionary modules can be distinguished from developmental modules (Wagner et al. 2007). The latter could provide the basis for a general theory of development, and have received special attention in the field of evolutionary developmental biology (evo-devo) (Raff 1996, von Dassow \& Munro 1999, Bolker 2000). Other authors contrast morphological, evolutionary, and developmental modules (Callebaut 2005). Yet another classification scheme segregates structural from developmental and physiological modules (Winther 2001). In this thicket of classifications (Wimsatt 2007), it is difficult to still see the forest for the trees.

Let us therefore remember that we are interested in the modularity of the genotype-phenotype map and how it relates to phenotypic evolution. Our aim is to explain the modular structure of the genotype-phenotype map in causal-mechanistic terms. Thus, we focus on functional modules, or quasi-independent subsystems of the epigenotype, which are responsible for the generation of specific morphogenetic patterns or phenotypic traits (Fig. 1). We must avoid falling into the trap of a "transcendental argument" by simply postulating such modules (Brandon 1999). Functional modules have to be identified and characterised empirically. This is not an easy task. It not only requires functional decomposition, but also reconstitution of the integrated activity of modules by systems-level approaches. We review such approaches that use structural and regulatory criteria as proxies for functional ones. We discuss the severe limitations inherent in these criteria. This motivates us to introduce a novel type of module, the dynamical module, which is empirically identifiable in a wide range of developmental processes and intimately related to function. We discuss methods to characterise such modules in formal

4. As a simple example of what is meant here, let us consider the understanding of social behaviour in terms of interactions between individuals. In such a description, each individual is a module-it has tight internal cohesion, with intrinsically-determined principles, and relative independence. However, sociallevel behaviour emerges from the interaction between modules (individuals), so independence is necessarily limited. In this familiar context, there is clear explanatory benefit in representing the population as a collection of quasi-independent individuals, even though one could, in principle, argue that population-level dynamics are "nothing more than" the dynamics of a collective physiology. This provides a simple example of what Simon $(1962,1973)$ means by near-decomposability, illustrating its evident utility. Note that in this picture, individuals can be thought of as structural, functional, and variational modules, depending on the perspective being taken, and the question being addressed. 
models and actual developmental systems. We briefly survey known dynamical modules in evolution and development. We conclude that dynamical modules provide a powerful new approach for the causal-mechanistic decomposition of the dynamic and evolvable structure of the genotype-phenotype map, and argue that dynamical modularity can be seen as the conceptual foundation for a new research programme and theoretical perspective on developmental evolution.

\section{Functional modules}

Our aim is a functional decomposition of the genotype-phenotype map, because it is the only kind of decomposition that allows us to directly link evolving generative processes to evolving morphological traits (Fig. 1). In this sense, we agree with Hartwell et al. (1999, p. C47), who argue that functional modules are a "critical level of biological organization." These authors define a functional module as "a discrete entity whose function is separable from those of other modules." (ibid. p. C48). Similarly, Mireles and Conrad (2018, p. 1) define a functional module as consisting of "sets of elements that act together in performing some discrete physiological function." These definitions are as concise as they are unworkable since they leave many crucial questions open. How do we define a function? What kind of discrete entity are we looking for? And how do we identify such an entity in the context of a complex regulatory system ${ }^{5}$ ?

For our present purposes, we adopt Cummins' (1975) systems-oriented view of biological functions. For our functional decomposition, we need to know which components of a regulatory system have the capacity to contribute to a given feature of the whole ${ }^{6}$. This contribution is their function.

What kinds of system components are we after? A common reductive approach seeks a decomposition of the generative processes comprising the genotype-phenotype map into a network of interacting elemental regulatory factors. These factors can (but do not have to) be genes and their products. In this context, identifying modular subsystems means identifying subnetworks (also called subcircuits) that contribute to a specific function. This approach goes back to Bonner (1988) who first proposed "gene nets" as discrete units of development.

5. The difficulty of the problem can be illustrated with the following example: it is notoriously hard to formally define the separable function of a "left back" in a football team, even if we have a clear notion of what the role implies in practice. See https://en.wikipedia.org/wiki/Association football positions for a good description of a complex network with adaptive functional modules.

6. Other accounts of function in biology are the etiological account by Wright (1973), which is more suitable to answer the question of why a given function evolved, or the organisational account (e.g. Christensen \& Bickhard 2002, Mossio et al. 2009) which refines Cummins' framework by assessing how a component of a living system contributes to its self-maintenance and self-production. 


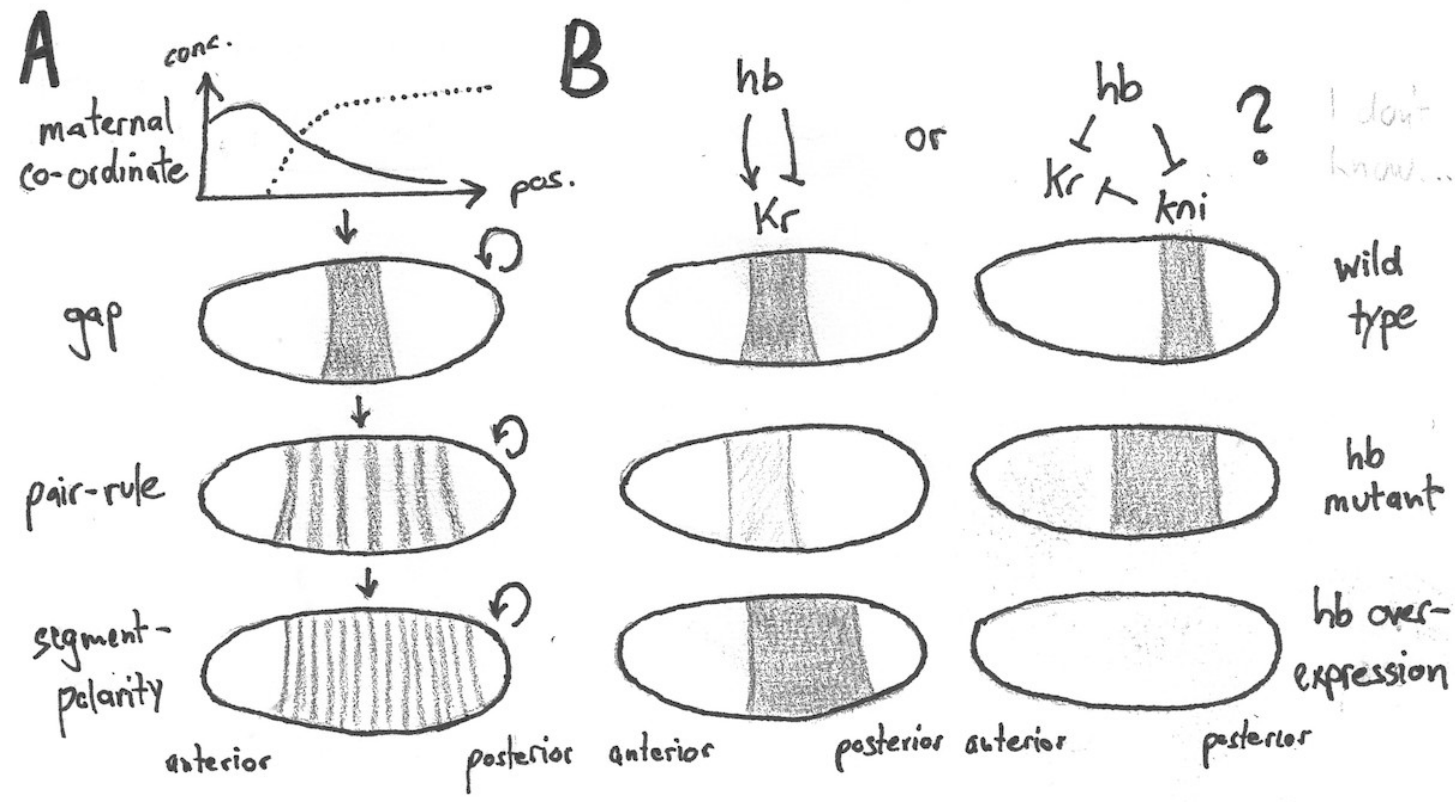

Figure 2: The tools of genetics and molecular biology establish the necessity, but never the sufficiency, of components and their interactions for a functional trait. (A) The functional role of the segmentation gene network in the vinegar fly Drosophila melanogaster is to subdivide the body plan into 14 segmental units. The segmentation gene network exhibits a complex hierarchical regulatory structure: protein gradients encoded by maternal-coordinate genes activate gap genes which, in turn, activate pair-rule and segment-polarity genes. Lower levels in the hierarchy are regulated by, but do not regulate, genes at higher levels. There is extensive cross-regulation within each layer. (B) The exact structure of a regulatory network cannot be established by genetic and molecular approaches alone. As an example, we show experiments which aim to establish the regulatory effect of hunchback ( $h b)$ on Krüppel (Kr). The expression patterns of $K r$ in $h b$ loss-of-function mutants and mutants with $h b$ overexpression are ambiguous in this regard. Anterior expansion of the $K r$ domain in the mutant, and posterior displacement of its anterior domain boundary upon over-expression indicate that $h b$ has a repressive effect on $K r$. At the same time, $K r$ is weakened in the mutant, and expands posteriorly upon over-expression, which suggests an activating effect. This could either be explained by different regulatory effects at different concentrations of $\mathrm{Hb}$ protein, or by an indirect activation via knirps (kni), which is repressed by $h b$ while being a repressor of $K r$. Which network topology is the correct one cannot be established without additional evidence from quantitative and integrative approaches. See Jaeger et al. 2004b for discussion; Jaeger 2011 for references to the primary literature.

The methods of molecular biology and genetics provide powerful experimental tools to map individual genes, their products, and their interactions, to specific functions ${ }^{7}$. For instance, Nüsslein-Volhard and Wieschaus (1980) performed a mutagenesis screen that saturated the genome with mutational hits to identify the set of genes involved in the determination of body segments in the early embryo of the vinegar fly Drosophila melanogaster (Fig. 2A). Interactions between these genes were then characterised by years of careful and systematic genetic and molecular experimentation (e.g., Akam 1987, Ingham 1988). All of these experiments use

7. We will focus on genetic and molecular approaches to functional modularity here. An alternative, more formal and top-down, approach based on "phenotypic building blocks" has been proposed by Mireles and Conrad (2018). 
perturbation assays to determine the contribution of a factor (or an interaction) to segment determination. This research programme led to many revolutionary new insights into the genetic basis of development ${ }^{8}$. But did it also yield a satisfactory characterisation of the segment-determination module?

The answer to this question is a clear and resounding no. Modules are not only dissociable from other modules, but are also characterised by their internal organisation. The components within a module interact, often in complex ways involving regulatory feedback, to generate the activity that defines the module's function. Reductionist experimental approaches for mechanistic decomposition (which attempt to infer pairwise regulatory interactions) are not best suited to the task of reconstructing an integrated module from its genetic or molecular components. They can only establish that a set of regulatory factors is necessary, but not that it is also sufficient, to account for a given function. To make things worse, it is impossible to unambiguously establish the nature and strength of regulatory interactions based on qualitative genetic evidence alone (Fig. 2B) (this issue is discussed, at some length, in the Introduction of Jaeger et al. 2004b). These limitations are fundamental, not merely practical (Isalan and Morrison 2009, Isalan 2009), but can be overcome with complementary systems-level and integrative criteria for the definition and identification of functional modules.

We illustrate these difficulties using the example of character-identity networks (ChINs) (Wagner, 2007, 2014). ChINs are subnetworks whose function it is to determine the character identity of specific traits. Character identity captures the continuity of a trait in an evolutionary lineage, independent of its precise structure or function. It contrasts with character state, which is defined by the specific size, shape, or color of a character. For example, "insect forewing" and "hindwing" are character identities, while "wing blade," "haltere," and "elytra," represent different states of these characters (Fig. 3). Being able to identify functional modules responsible for character identity would yield a mechanistic explanation for homology (Wagner 2007, 2014). However, the concept of a ChIN is problematic for the general reasons outlined above: the sufficiency of identified components and the precise structure of the subnetwork cannot be established by genetic means alone. In addition, it is rarely easy to pinpoint factors that exclusively contribute to the identity, but not the state, of a trait. The same gene can contribute to both aspects. Moreover, definitions of character identity and state are fluid and context-dependent. Finally, different genes and interactions may be involved in generating homologous trait identities in different lineages due to network drift (Weiss \& Fullerton 2000, True \& Haag 2001, Wagner 2011). All these issues highlight that there is a fundamental problem of correspondence between this type of genetic network and the functional traits they are supposed to explain, which is "neither trivial nor presently soluble" (von Dassow \& Munro 1999 , p. 317). On top of it all, we will encounter an additional issue with explanations in terms of static network structure in the next section. Taken together, this means that ChINs in particular, and networks based on genetic and molecular decomposition in general, only provide a very rough first sketch of a true decomposition of the genotype-phenotype map in

8. Recognized by Nobel Prizes for Janni Nüsslein-Volhard and Eric Wieschaus in 1995. 
terms of functional modularity ${ }^{9}$. In the following sections, we look at different approaches that aim to improve the quality of this sketch.

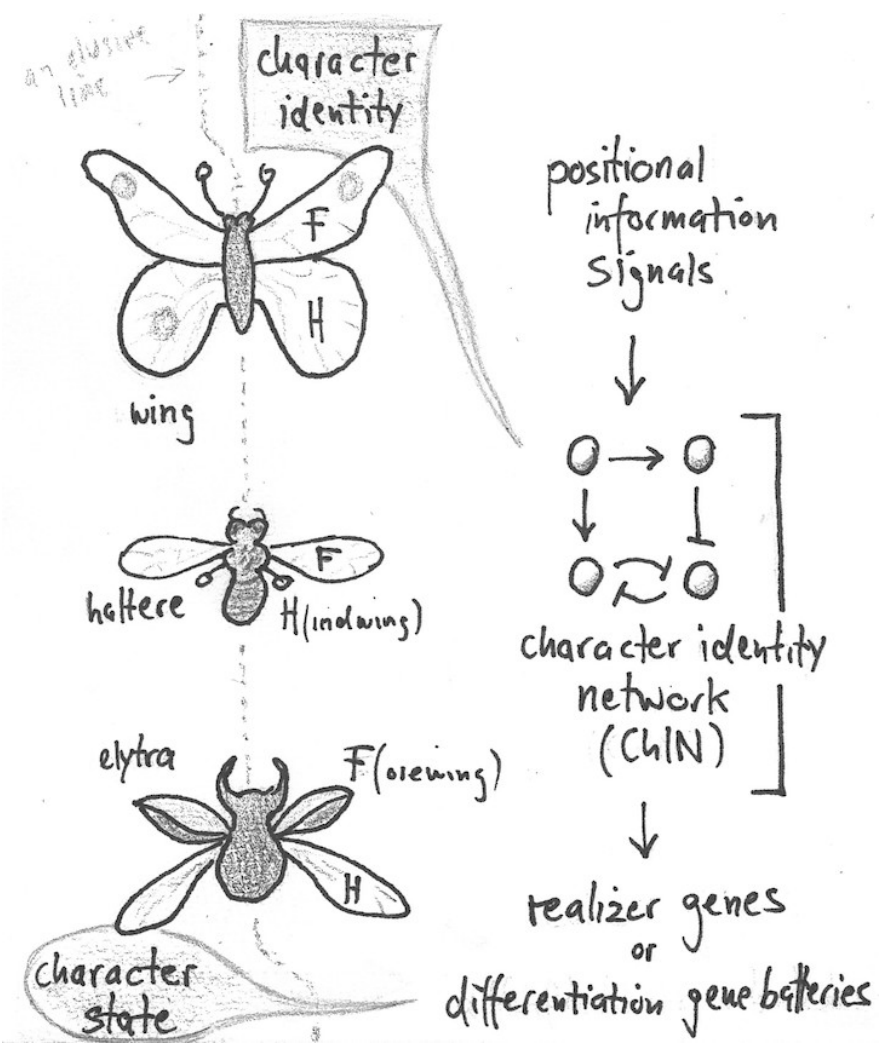

Figure 3: Character identity neworks (ChINs) determine the identity, but not the state, of a character trait. Insect wings are shown as an example. Fore-/hindwing: character identity. Morphology-wing, haltere, elytra: character state. ChINs are activated by general positional information signals, and then activate realizer genes (or differentiation gene batteries) that determine character state. After Wagner (2007).

\section{Structural modules}

We have argued in the last section that genetic decomposition needs to be complemented with systems-level criteria to arrive at a satisfactory characterisation of an integrated functional module. The most common approach is to use features of local network structure (or topology) to identify subsystems. The idea is that the internal cohesion of modules is reflected in the type and density of interactions among components within and between subnetworks. We will call modules identified in this way structural modules. This network-based definition is not to be

9. The problems raised here, and their relation to causal-mechanistic explanation in evolution and development, are discussed in detail in DiFrisco \& Jaeger (2019). Moreover, DiFrisco et al. (2020) specifically address the problem of correspondence between genetic networks and functional traits by introducing the concept of a character identity mechanism (ChIM), which accommodates non-genetic components and interactions, multi-level causation, and network drift. 
confused with modules that consist of actual physical structures such as protein complexes (Winther 2001).

We can use informal arguments or formal algorithms to identify structural modules. A prominent example of an informal approach relies on computer metaphors to characterise the developmental and evolutionary roles of subsystems in gene regulatory networks. Through decades of sustained and painstaking genetic and molecular analysis, Eric Davidson and colleagues have characterised a large regulatory network responsible for the specification of the endomesoderm and its embryonic derivatives during early development in the sea urchin Stronglyocentrotus purpuratus (Davidson et al. 2002, Oliveri \& Davidson 2004). This network can be divided into a hierarchy of subcircuits. The authors use an ad hoc combination of functional criteria as a first step to achieve this. Genes are assigned to subcircuits depending on their temporal expression profile (see "Regulatory modules" below), the specific function they contribute to (e.g. endo- vs. mesoderm specification), and/or the kind of transcription factors that bind to the regulatory sequences that govern their expression (Oliveri \& Davidson 2004, Levine \& Davidson 2005).

The resulting set of subcircuits are then classified into kernels, plug-ins, and differentiation gene batteries connected by input-output switches (Fig. 4A) (Davidson \& Erwin 2006, Erwin \& Davidson 2009). Kernels are highly conserved subcircuits involved in the specification of fundamental features of the body plan. Plug-ins are also conserved to some extent, but contribute to different developmental processes in different lineages. Gene batteries comprise the downstream effector factors responsible for cell differentiation and morphology. Switches represent connections between modules. Mutational effects depend on where in this modular hierarchy a mutation occurs: micro-evolution is driven by changes in differentiation gene batteries, body plan evolution at higher levels (Davidson 2011). In particular, plug-ins can be co-opted into new functions by rewiring the switches that connect them (Davidson \& Erwin 2006). Kernels, plug-ins, and gene batteries can be further subdivided into a set of stereotypical minimal regulatory motifs (implementing positive or negative feedback loops, for example), whose functions are supposed to be derivable from their structure (Davidson 2010, 2011, Peter \& Davidson 2011, 2017). In this analysis, structural arguments supplement functional criteria for the classification of subcircuits and the identification of low-level regulatory motifs. 


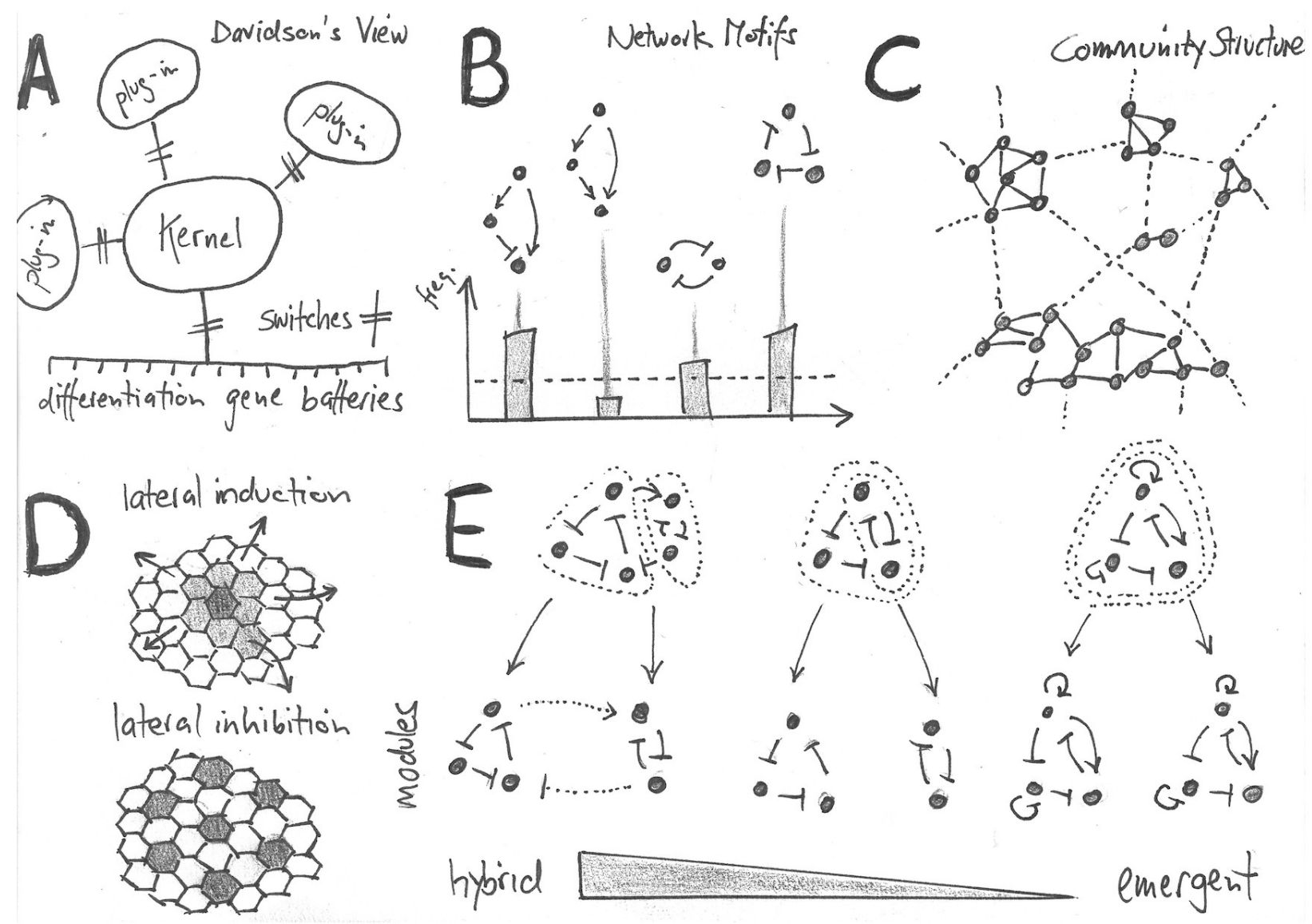

Figure 4: Structural modularity and its problems. (A) Eric Davidson and colleagues divide complex networks into modules that represent conserved kernels, plug-ins (can be co-opted during evolution), and differentiation gene batteries, all connected by switches. Mutational effects depend on where in this hierarchy they occur. (B) Network motifs are small networks with a given regulatory structure. They are identified by their enrichment in complex regulatory networks (represented by histogram, expected motif frequency shown as a dashed line). (C) Community structure identifies modules by detecting differences in the density of interactions within and between them. (D,E) Study of multi-functional circuits by Jiménez et al. (2017): (D) shows the two functions that each circuit must perform. Lateral induction propagates a signal from its source across a tissue; lateral inhibition leads to a salt-and-pepper pattern. (E) Structural modularity in multi-functional circuits occurs along a spectrum: hybrid circuits are composed of completely disjoint modules for each function; emergent circuits show overlap between modules. In extreme cases, the modules performing each function are completely identical to each other. Hybrid circuits are an exception, emergent circuits the norm. See text for details.

In addition to such pragmatic approaches, formal methods identify modules based on local features of network structure. We briefly describe two prominent methods that use complementary aspects of the definition of a module. Network motifs are small subcircuits with a specific internal regulatory structure that are detected through their statistical enrichment ${ }^{10}$ in large, often genome-wide, regulatory networks (Fig. 4B) (Shen-Orr et al. 2002; Milo et al. 2002,

10. A subnetwork with a given topology is considered as statistically enriched if it occurs more frequently in a given network than in a randomly-connected network with equivalent global properties. 
Alon 2006, 2007). This approach is based on enrichment indicating functional relevance. It assumes that the behaviour of the whole network can be understood as a composite of the well-characterised dynamics of its component motifs. At the very least, we are supposed to learn something about network function from the statistical distribution of motif frequencies (Milo et al. 2004, Shellman et al. 2013). In contrast, algorithms that detect community structure in network graphs rely on differences in the local density of network connections (Girvan \& Newman 2002, Radicchi et al. 2004, Newman 2006, Newman et al. 2006, Fortunato 2010). This approach posits that nodes within a module "are joined together in tightly knit groups, between which there are only looser connections" (Fig. 4C) (Girvan \& Newman 2002, p. 7821). On this view, structural modules correspond to "cliques" of densely connected network nodes (Alexander et al. 2009). While network motifs are allowed to overlap, structural modules defined by community structure usually consist of disjoint sets of nodes, although some algorithms yield overlapping subcircuits as well (Palla et al. 2005). The two approaches can be combined to find community structures of clustered network motifs (Benson et al. 2016).

As a means of exploring functional modularity, structural approaches have shortcomings. The most obvious and consequential of these is that network structure constrains, but does not determine function. Even very simple networks, considered in isolation, can exhibit a range of qualitatively different dynamical behaviours depending on the kind and strength of their regulatory interactions and the stability of their components (Mangan \& Alon 2003, Wall et al. 2005, Ingram et al. 2006, Siegal et al. 2007, Payne \& Wagner 2015, Ahnert \& Fink 2016, PerezCarrasco et al. 2018, Page \& Perez-Carrasco 2018). In addition, network motifs can be extremely sensitive to their temporal (Rosenfeld \& Alon, 2003, Kim et al. 2011) and network context (Kashtan et al. 2004, Dobrin et al. 2004, Mazurie et al. 2004, Ingram et al. 2006, Solé \& Valverde 2006, Burda et al. 2011, Benson et al. 2016, Gorochowski et al. 2018). The addition of a single regulatory interaction can completely change the the range of different dynamical behaviours a network can implement (Perez-Carrasco et al. 2018, Verd et al. 2019). Indeed, such behavioural diversity of regulatory networks is to be expected, and is central in generating adaptive dynamics on developmental, physiological, and evolutionary scales. We therefore cannot assume a network's dynamics to be a straightforward composite of the behaviour of its modular components. This is illustrated by a series of studies that examined segmentation gene expression in Drosophila in terms of network motifs. Despite claims to the contrary, none of these models were able to assign specific patterning functions to motifs, or recover the correct temporal dynamics of the whole system (Zinzen \& Papatsenko 2007, Ishihara \& Shibata 2008, Papatsenko 2009). Indeed, we are left with a massive explanatory gap between the characterisation of structural modules and their biological function. Any approach that presupposes a close connection between structural and functional modularity in evolved systems is doomed to fail ${ }^{11}$. It is quite telling that even the most stalwart defenders of the idea that structure determines function must rely on dynamical models to understand the behaviour of their network (Peter et al., 2012, Peter \& Davidson, 2017).

11. This is in contrast to many engineered systems, which are designed and constructed specifically to maintain simple and predictable relationships between structural and functional modularity. Such simplicity is appealing, but cannot be expected to hold in naturally evolved networks. 
A second limitation is that many regulatory networks are not structurally modular, even though they are evolvable (e.g. Crombach \& Hogeweg 2008) and exhibit functional modularity (Alexander et al. 2009). This is beautifully illustrated by an in silico screen that searched for small networks with multi-functional behaviour (Jiménez et al. 2017). The screen proceeded in two steps: first, it identified topologies of minimal networks with intercellular signaling that generate both lateral inhibition and lateral induction depending on the strength of regulatory interactions (Fig. 4D). Second, they filtered the selected circuits for networks that can perform both functions in the presence or absence of an external signal. The results indicate two distinct kinds of multi-functional circuits (Fig. 4E). Hybrid circuits, on the one hand, are the sum of their mono-functional structural modules: they use disjoint sets of nodes for each function; emergent circuits, on the other hand, show overlap between the nodes of different modules ${ }^{12}$. Most emergent circuits are only partially modular in the structural sense and, in the most extreme case, the structure of both functional modules completely coincide (Fig. 4E). Many multi-functional circuits are emergent, and therefore only functionally, but not structurally dissociable. Methods based on structural modules will fail for these circuits.

Finally, there is a fundamental conceptual problem with explanations in terms of static network graphs that is essential for our argument. Static graphs cannot, in principle, be causalmechanistic explanations, because they are not extended in time (Jaeger \& Monk 2015, Nicholson \& Dupré, 2018, DiFrisco \& Jaeger 2019). They cannot explain how the network progresses from initial conditions to output. Causality and mechanism must be embedded in time. Modules must persist to exert an effect (Callebaut 2005). This requires us to search for explanations in terms of the dynamics of modular systems. Dynamic modularity, however, poses its own challenges, since process modules are much more ephemeral than structural ones (Bolker 2000).

\section{Regulatory modules}

Probably the simplest way to identify dynamic modules in a gene regulatory network is to look for factors with correlated or anti-correlated temporal expression profiles (Eisen et al. 1998). Such co-expression clusters or synexpression groups are called regulatory modules ${ }^{13}$ (Tavazoie et al. 1999, Segal et al. 2003, Bar-Joseph et al. 2003, Babu et al. 2004, Niehrs, 2004). Genes known to be functionally related have products whose expression profiles tend to cluster together (Eisen et al. 1998, Tavazoie et al. 1999). Inversely, evolutionary conservation of regulatory modules can be used as indicator for functional relationships (Teichmann \& Babu

12. The emergent networks of Jiménez et al. (2017) should not be confused with emergent networks as defined earlier by Salazar-Ciudad et al. (2000, 2001a,b). While "emergent" sensu Jiménez indicates heavy overlap between functional modules, "emergent" sensu Salazar-Ciudad indicates a flat network structure rich in regulatory feedback, which is contrasted to "hierarchical" networks with a more layered and feed-forward topology.

13. Segal et al. (2003) also call regulatory modules "module networks." The terms are equivalent. Since it is easy to confuse module networks with structural networks, we will not use the term here. 
2002, Stuart et al., 2003). Co-expressed genes often share common regulators, which provides an additional criterion for module identification (Segal et al. 2003, Bar-Joseph et al. 2003). This approach scales well to large networks, up to genome scale (Babu et al. 2004, Bolouri 2014). For our purposes, however, it is limited in two important ways.

One problem is that the resulting network models are probabilistic and not causal-mechanistic (e.g., Bolouri 2014). This approach cannot move beyond correlational evidence to predict specific mechanistic interactions. Regulatory modules are purely statistical entities. The other problem with regulatory modules is that they are too simplistic. The underlying assumption is that regulation is direct and straightforward: correlated genes are co-activated, anti-correlated factors repress each other. Both cases can be corroborated by finding binding sites for the shared regulator. This should work well for differentiation gene batteries, which are by definition co-regulated and co-expressed (see "Structural modules" above). It will fail, however, to correctly circumscribe more complex upstream regulatory networks involved in orchestrating cellular and developmental processes. As soon as more than two or three factors are involved in the non-linear regulatory structure of such a network, or as soon as the system is distributed over a spatially differentiated context, indirect effects can create mechanistic interactions resulting in complex patterns beyond simple correlations. Approaches based on correlated co-expression will fail to identify such links.

\section{Dynamical modules: definition and detection}

Since our aim is to understand functional organisation, we are interested in the full set of dynamics associated with a network architecture. Our starting point is therefore this full set of dynamics, rather than the structure of the network, as discussed above. We describe the dynamics of a network in terms of temporal sequences of states, which define the system's trajectories. The space of all possible trajectories-a network's phase space-has its own characteristic structure, and it is this structure that we seek to interrogate (Strogatz, 2014). A central role is played by attractors: specific state sequences that trajectories approach asymptotically (Jaeger and Monk, 2014). These represent the possible long-term behaviours of the network. Approaches that explore the structure of phase space and its constituent attractors lead to the notion of dynamical modules.

Dynamical modules (also called dynamical subsystems) ${ }^{14}$ provide a powerful alternative to structural and regulatory modules. The idea of dynamical modularity first appears implicitly but clearly in Stuart Kauffman's "Origins of Order" (1993). Kauffman's earlier work on ensembles of Boolean network models (Kauffman, 1969, 1974, 1987) had revealed that such systems could be in an ordered or "chaotic" state. "Chaotic" systems, in this context, do not settle into any stable or repeating state within a realistic amount of time, and easily diverge from their original

14. Alexander et al. (2009) use the term "activity motifs" for dynamical modules as defined here. We find this potentially confusing due to its similarity with "network motifs" (Alon, 2007). Unlike network motifs, dynamical modules are not defined structurally or in terms of their enrichment within a network. 
attractor upon perturbation. In contrast, ordered systems exhibit few short attractor cycles and tend to return to their original state after a majority of small state perturbations.

Kauffman (1993) suggests that natural selection drives evolving networks into the border region of the ordered regime (the "edge of chaos"). It does this by altering the topology or density of regulatory interactions or the kind of logical functions that are present in the network. Systems at the edge of chaos contain a large connected component of nodes with ordered dynamics, which percolates around "islands" of chaos. Only such networks exhibit behaviour that is complex, yet controllable, just like biological systems. Perturbations do not travel widely across components, but rather accumulate in the chaotic island they occurred in. Other islands can accumulate their own perturbations relatively independently. Obviously, Kauffman's notion of a "chaotic island" corresponds to some kind of dynamical module of the overall network.

A few years later, von Dassow \& Munro (1999) defined a module as a developmental subsystem manifesting quasi-autonomous behaviour. This definition is close to what we mean by a dynamical module here, but is still too vague. A more explicit argument appears in von Dassow and Meir (2004), which states that connectivity is not sufficient: modules are units with their own intrinsic dynamics. An even more specific definition was given by Irons and Monk (2007, pp. 1/2): "Rather than being a protein complex or group of co-expressed genes, [a dynamical] module can be viewed as the temporal activity (dynamics) of a group of genes/proteins that controls a specific function in different environmental conditions, cell types and/or tissues. For example, the temporal activity of genes/proteins controlling progression through the cell cycle (in many different environmental conditions and cell types) can be viewed as a module" (see also Irons 2009). Of course, this definition can be extended to subsystem components that are neither genes nor proteins. Put slightly differently, dynamical modules consist of the activities of a set of network nodes, connected by regulatory interactions, that implement a particular type of behaviour exhibited by the larger network they are embedded in (Verd et al. 2019). In the words of Benítez \& Alvarez-Buylla (2010 p. 11), such a module is based on a "set of nodes and interactions that, being part of a larger network, may exhibit a dynamic trait (e.g. a certain number of attractors) in a semi-autonomous way" (see also Benítez et al. 2008, 2011). Dynamical modules can be derived from time-series data (Alexander et al. 2009) or from dynamical computational models (Irons and Monk, 2007, Siebert 2009, 2011, Verd et al. 2019). They interact in a nested and hierarchical way to generate the overall dynamical repertoire of a complex regulatory network.

Dynamical modules also appear in the study of evolution. Newman and Bhat $(2008$, p. 2) define a dynamic patterning module as "a set of molecules produced in a cluster of cells, along with one or more physical effects mobilized by these molecules so as to generate an aspect or alteration in the cluster's form or pattern" (see also Newman and Bhat 2009; HernándezHernández et al. 2012). Dynamic patterning modules are used to explain the role of selforganisation in the evolution of early multicellular organisms and more recent developmental processes. The emphasis here is on the interaction of regulatory networks (the "set of molecules") with their cellular and tissue context. Nevertheless, dynamic patterning modules 
are developmental subsystems that are defined in terms of their dynamic behaviour. In this sense, they are a subtype of dynamical module.

Dynamical modules are not as easy to detect as structural or regulatory modules. To identify them, it is necessary to find patterns in the system dynamics that are present as parts of a wide range of different behaviours of the whole system. They typically do not correspond to clusters in network topology or expression dynamics. The dynamical behaviour they generate can be complex, and assumes no simple correlation between the expression profiles of module components. The fact that dynamical modules are features of the whole dynamical repertoire of a system is what makes their detection challenging. Irons and Monk (2007) introduce an algorithm to identify dynamical modules given a set of discrete-state, discretetime attractors. These attractor states can be generated by a Boolean dynamical model, or directly identified from experimental data. They capture the non-transient dynamical repertoire of a system. Irons and Monk (2007) use a simple toy model to introduce the method. A brief illustration of the approach will give us a precise understanding of what a dynamical module is.

Boolean networks consist of nodes and their interactions (Fig. 5A). At a given time, each node can either be off (state 0 ) or on (1). The interactions between nodes are defined by logical tables that connect all possible combinations of inputs to a particular node with specific output states (Fig. 5B). Starting from a given initial state, the system will go through a defined succession of transient states until it reaches a stationary or cyclical attractor. A Boolean network has a finite number of possible states, and so has the advantage that the full repertoire of attractors (and the transient state sequences leading to them) can be enumerated. In this way, one can generate the full set of attractor states, which are the starting point for the identification of dynamical modules. Our toy model has four distinct attractors, three of them cyclical, one stationary (Fig. 5C).

Given the set of attractors, the algorithm identifies subsets of nodes in the network that exhibit a characteristic dynamic (i.e. time-sequence of states) that is conserved in multiple attractors (Irons and Monk, 2007). To achieve this, it examines partial states (states of a subset of nodes in the system) and their state sequences, which are ordered sets of partial states in time. A partial state sequence occurs in an attractor, if its partial states remain constant or continually cycle within an attractor and there is no smaller partial state sequence that does the same. Based on this, the algorithm finds a set of subsystems, which are maximal, non-overlapping partial state sequences that occur within the given set of attractors (Fig. 5D). Each subsystem generates a typical dynamical behaviour. Put together, they compose the overall behaviour of the whole network. It is important to emphasise that subsystems often overlap in that they share nodes and interactions between each other. Furthermore, subsystems can occur in different combinations in multiple attractors. Therefore, neither their network graphs nor their dynamical functions need be disjoint. Dynamical modules not only can, but are expected to show overlap in both structure and function. 

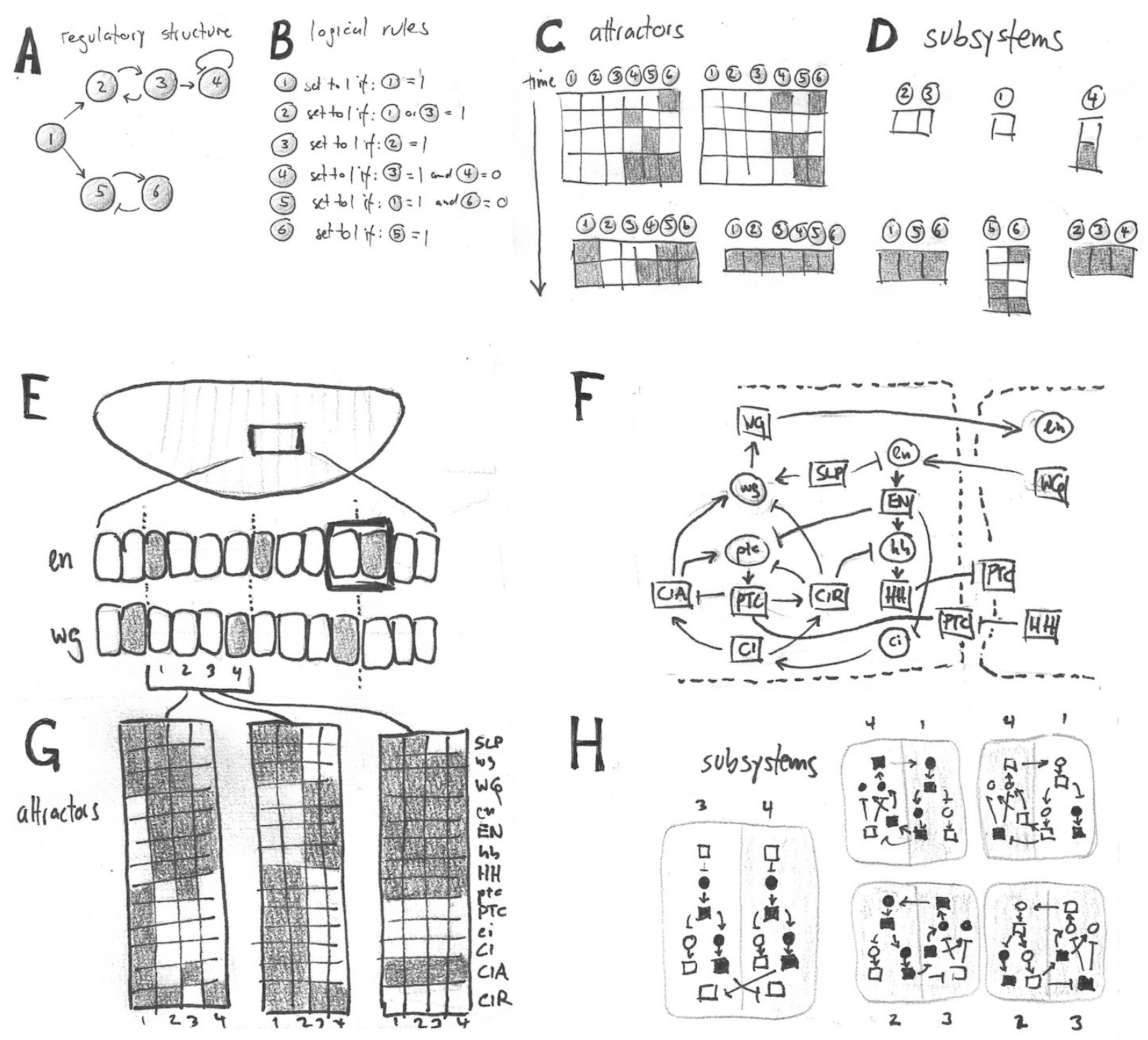

Figure 5: Dynamical modules in Boolean network models. (A-D) Toy model, illustrating the algorithmic detection of dynamical modules. Regulatory structure $(A)$ is given by logical rules $(B)$ that determine the interactions between nodes. This results in multi-stable dynamics with four attractors, shown as temporal sequences of states of the system (C). Dynamical modules correspond to a set of subsystems that provide an optimal decomposition of the attractors (D). The attractors are simple composites of these subsystems. (E-H) Application to the segment polarity system of Drosophila melanogaster (see Fig. 2A). (E) Segment polarity genes produce an alternating pattern of en and wg stripes across four cells within a segmental unit in the embryo. (F) Regulatory structure of the segment polarity network across two cells (indicated by dashed lines). Activating interactions are shown by arrows, repressive interactions by T-bars. (G) The system contains three attractors that correspond to empirically observed stable expression patterns (shown across all four cells of a segmental unit). (H) Attractors can be decomposed into subsystems as in (C) and (D). Five main subsystems explain most of the dynamics within the attractors shown in (G). These subsystems consist of intercellular feedback circuits that show symmetries across several different cell boundaries. See text for further detail. After Irons \& Monk (2007). 
In addition to identifying modular subsystems, the algorithm can be used to examine the hierarchical interactions among subsystems. A subsystem is hierarchically linked to another subsystem if its occurrence entails the occurrence of the other in an attractor. It is said to trigger the other subsystem. By measuring the percentage of perturbations within a subsystem that return to the original attractor, we can assess the robustness of the whole network, as well as the internal and external robustness of each module, that is, its response to internal or external perturbations.

Irons and Monk (2007) illustrate the biological application of their method using a wellestablished Boolean model of the segment polarity gene regulatory network of Drosophila melanogaster (Albert \& Othmer 2003; Chaves et al 2005). The model consists of a regulatory network with 13 nodes representing interactions between transcription factors and cell-cell signalling factors, which are distributed over a parasegmental unit represented by a spatial domain of four cells (Fig. 5E, F). This network shows no obvious structural modularity. The system has ten attractors, only three of which correspond to observed gene expression patterns in the embryo (Fig. 5G). Detection of dynamical modules yields a total of 19 subsystems, five of which capture a large proportion of the system's global dynamics (Fig. $5 \mathrm{H})$. These dynamical modules correspond to intercellular variants of core subsystems for segmentpolarity patterning that were identified in an earlier in silico screen for robustness in continuous models of three-node networks (Ma et al. 2006). While that screen was based on ad hoc assumptions about the functional core of the network, the algorithm devised by Irons and Monk (2007) provides an unbiased identification of dynamical modules. Analysis of the hierarchical relationships between subsystems revealed a symmetry in the system centered on the border between the first and the second cell of the parasegment: every subsystem in cells one and four has a counterpart in cells two and three with analogous regulation (Fig. 5H). SLP (Fig. 5F) can be identified as the regulatory factor responsible for setting up this symmetrical pattern. The example highlights how a network that is not structurally modular can be subdivided into dynamical modules, and how the analysis of these modules explains the functionality, that is, the switch-like bistable behaviour of the whole system. The algorithm was also applied to a Boolean model of the yeast cell cycle (Irons 2009).

\section{Dynamical modules: gradients, gap genes, and the AC/DC circuit}

The algorithm for detection of dynamical modules developed by Irons and Monk applies to networks with discrete state spaces. While discrete state models are appropriate for some systems, continuous state models (e.g. differential equations) are preferable for processes involving gradual changes in regulator concentration. Any generalisation of the algorithm to continuous states must overcome two difficulties. First, information on multiple attractors of the system under study is required to define dynamical modules. Second, a suitable measure of "equivalence" of dynamics across attractors replaces the requirement that the dynamics of the module are strictly conserved across sets of attractors. Patterning by morphogen gradients provides many examples of gradual processes that require a continuous approach (see 
Fig. 2A, top panel) (Wolpert 1968, 1969; Jaeger et al. 2008; Briscoe \& Small 2015). In these systems, target gene activation depends on the spatio-temporal concentration distribution of the morphogen. Boolean models with discrete on/off states are not suitable in this context. Can the notion of dynamical modularity be extended to such continuous systems?

One particularly well-studied network that drives morphogen-based pattern formation is the gap gene network of Drosophila melanogaster (Jaeger 2011). It constitutes the top-most regulatory layer of the segmentation gene hierarchy we discussed in the section "Functional modules" (Fig. 2A). It is a relatively simple patterning system that operates in a syncytial embryo without growth or rearrangements of tissue geometry. Gap genes are activated by gradients of maternally expressed transcription factors; in addition, they auto-activate and cross-repress each other, which results in a staggered, overlapping arrangement of their expression domains (Fig. 6A). The study we present results from a research programme to reverse-engineer the gap gene system by fitting dynamic models of the network to quantitative spatio-temporal gene expression data (Jaeger \& Crombach, 2012, Crombach \& Jaeger, this volume). The topology of the gap gene network does not exhibit any structural modularity. And yet, the system implements two clearly distinguishable dynamical regimes in the anterior versus the posterior of the embryo: while anterior domain boundaries remain at a stationary position and are generated by switch-like multistability, posterior boundaries shift over time and are driven by an underlying damped oscillator (Fig. 6B) (Jaeger et al. 2004a,b; Manu et al. 2009; Ashyraliev et al. 2009; Crombach et al., 2012; Verd et al. 2017, 2018; Jaeger 2018). Between the two regimes, a bifurcation occurs (Manu et al. 2009, Gursky et al. 2011, Verd et al. $2017,2018)$. In the absence of a suitable algorithm, we took a pragmatic approach to partition a continuous differential-equation model of this system into dynamical modules.

Gap gene patterning does not require diffusion of gap gene products between nuclei (Jaeger et al. 2004a; Manu et al. 2009; Verd et al. 2017). Consequently, the system can be thought of as an ensemble of nucleus-specific gap gene networks, each with a different set of maternal inputs. This provides an ensemble of dynamical repertoires of the network which can be used to detect dynamical modules. Our dynamical decomposition of the gap gene system is based on the observation that patterning never relies on more than three out of four gap genes in any nucleus within the trunk region of the embryo (Verd et al. 2019). This fact can be established rigorously by simulating versions of the network in which one node or gene has been eliminated, and measuring the sensitivity of the system to such in silico knock-outs. This approach identifies three distinct subsystems that drive gene expression in the anterior, middle, and posterior of the embryo. These subsystems consist of overlapping sets of genes that all share the same network structure: the AC/DC subcircuit, which was first identified and characterised in the context of morphogen-based patterning in the vertebrate neural tube (Fig. 6C) (Panovska-Griffiths et al. 2013, Perez-Carrasco et al. 2018). AC/DC circuits are simple three-node networks capable of producing oscillations and switch-like behaviour, depending on the strength of their regulatory interactions (Panovska-Griffiths et al. 2013, Perez-Carrasco et al. 2018, Verd et al. 2019). 


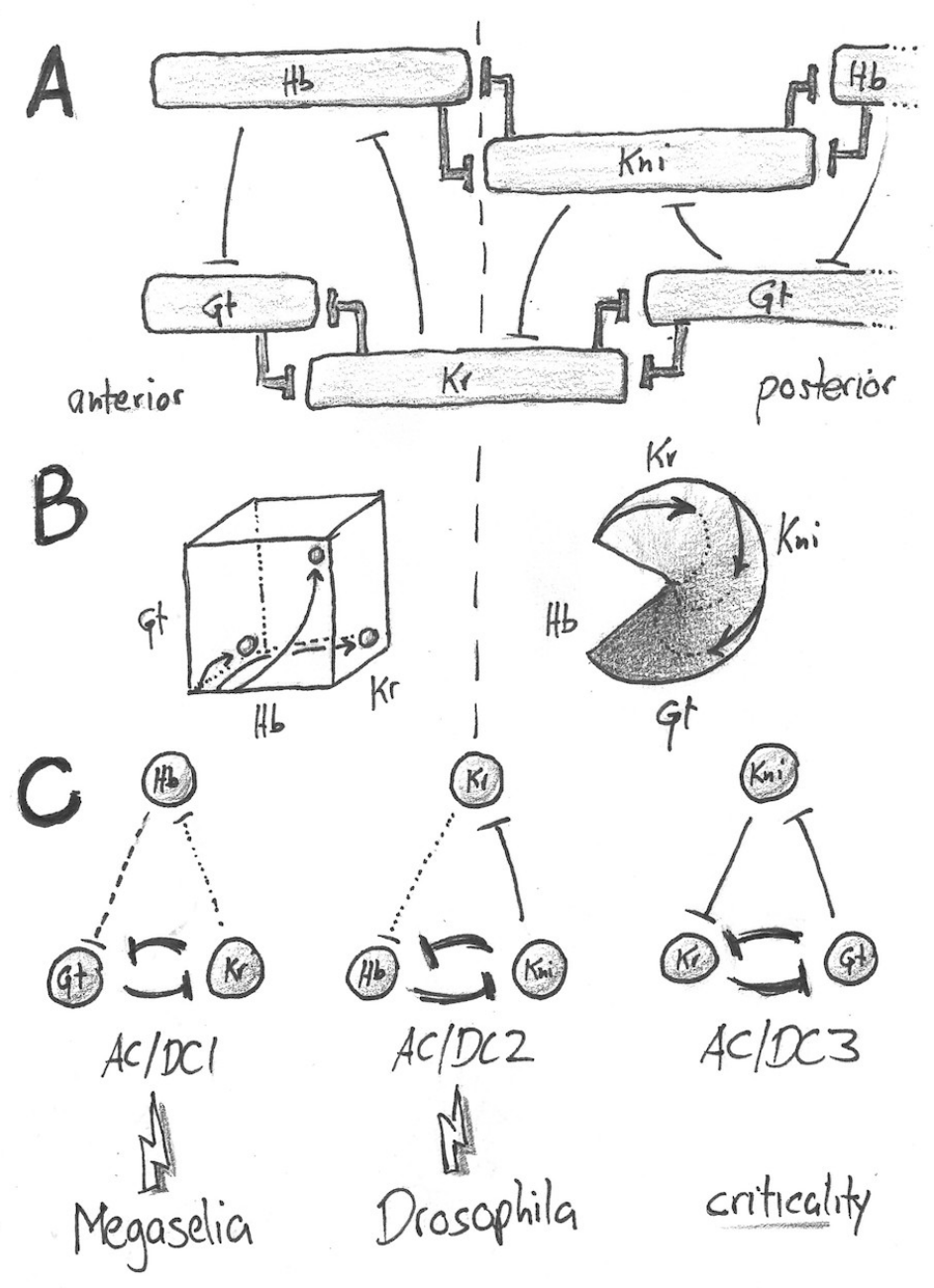

Figure 6: Modular decomposition of a continuous patterning system, the gap gene network in flies (see Fig. 2A). (A) Regulatory structure of the gap gene network. Boxes indicate spatial position of expression domains (anterior is to the left, posterior to the right). Repressive cross-regulation between gap genes is indicated by T-bars; activation by maternal gradients and gap gene auto-activation not shown. Two strong double negative (positive) feedback loops contrast with much weaker repression between overlapping gap gene domains. (B) The system implements two distinct dynamical regimes: in the anterior, domain boundaries are set by a multi-stable (switch-like) dynamic, in which the states of nuclei in different domains are converging towards different attractors (circles in box); in the posterior, nuclei cycle through a stereotypical succession of gap gene expression (driven by a damped oscillator) before all converging to the same attractor (indicated by circle with spiraling domains). Each nucleus starts from a different initial condition (dependent on maternal inputs) and thus traverses a different segment of the circle. This results in apparent (kinematic) shifts in the position of gap domains towards the anterior of the embryo, while domains in the anterior remain stationary over time. (C) The gap gene network can be decomposed into three AC/DC subsystems in the anterior, middle, and posterior of the embryo. There is extensive overlap in nodes and interactions between these subcircuits. AC/DC circuits reproduce the dynamics of the full system in their respective region of influence. Subcircuits are either structurally stable, or critical (exhibiting a bifurcation within their region of influence). AC/DC2 is critical in Drosophila, while AC/DC1 is critical in Megaselia (indicated by arrows). See text for details. After Verd et al. (2019). 
Next, we examine the dynamical behaviour of each subcircuit with phase space analysis (Strogatz 2014), and compare it with the dynamical repertoire of the whole gap gene system (Verd et al. 2019). This analysis reveals that each AC/DC circuit, in its own region of influence, is capable of driving the same type of dynamics as observed in the full system (Fig. 6B). $A C / D C 1$, in the anterior, generates expression domain boundaries through multistability: nuclei that express different gap genes find themselves in different basins of attraction due to receiving different maternal inputs. AC/DC3, in the posterior, implements a damped oscillator driving the stereotypical succession of gap gene expression that generates the kinematic shifts of domain boundaries over time. Since they drive a consistent dynamical regime across their regions of influence, these circuits are structurally stable. In the middle, AC/DC2 undergoes a bifurcation within its region of influence, just like the full model. It exhibits switch-like behavior anterior and damped oscillations posterior of the bifurcation boundary. The bifurcation indicates that this circuit is in a state of criticality, unlike the other two subsystems. The gap gene network is labile to change in some aspects, but robust towards others, which is analogous to Kauffman's notion of the system being at the "edge of chaos" (Kauffman 1993).

A number of points are worth noting about this type of dynamical decomposition. It is a topdown approach, just like the algorithm presented in the last section, using information on a range of different dynamical behaviours to partition the network into interlocking subsystems. Each AC/DC circuit implements a subset of behaviours of the full model. The behaviour of the full system corresponds to a straightforward combination of its modules. In this sense, AC/DC subcircuits fit perfectly into our definition of a dynamical module ${ }^{15}$. Each AC/DC circuit shares two out of three genes with its neighbour (Fig. 6C). This makes the gap gene network heavily emergent (Fig. 4E), which explains why it is not structurally dissociable. Surprisingly, AC/DC modules do not cleanly separate into switch-like or oscillatory behaviour. The bifurcation occurs within the region of influence of AC/DC2. In this regard, dynamical decomposition is not trivial, and requires careful mathematical analysis. In the absence of a formal approach, there are many valid ways to decompose a system dynamically. How we identify subsystems depends, to some extent, on our research question and assumptions. We illustrate this point by comparing our dynamical decomposition with a bottom-up in silico screen that identified core mechanisms for morphogen-based stripe (i.e. domain) formation.

Cotterell \& Sharpe (2010) used an approach equivalent to Jiménez et al. (2017) and Ma et al. (2006) (see also Ma et al. 2009) to search for minimal networks that produce a spatial stripe in a non-growing tissue in response to a static morphogen gradient. Their scenario resembles a simplified version of the gap gene system. Their screen yielded six distinct core mechanisms for stripe formation, which can be distinguished by their network topology and dynamical behaviour. Three of the six mechanisms were novel; they have never been observed in an

15. Note that for Boolean networks, dynamical modules have dynamics that are strictly conserved in multiple attractors; in the continuous-state gap gene system, each AC/DC circuit type corresponds to an equivalence class of dynamics. This provides an illustration of how we can adapt techniques developed in discrete-state systems to continuous-state systems. 
experimental setting. Out of the other three, two can be mapped onto the gap gene network. Surprisingly, the AC/DC circuit is not present, nor do any of the core mechanisms match a subset of interactions within the AC/DC topology. The explanation lies in the assumptions underlying the two different approaches to define subsystems. Dynamical decomposition requires the subsystems to produce well-defined spatial domains, and to reproduce the dynamical regimes observed in the full system. This requirement is much more stringent than the one used by Cotterell et al. and emphasises the importance of studying modules in their native network context.

\section{Dynamical modules everywhere}

We have argued that dynamical modules are more widespread in biological regulatory systems than structural ones. However, the only evidence we have presented so far stems from three isolated case studies: the gap gene network (Verd et al. 2019) and segment polarity network (Irons and Monk 2007) of Drosophila, as well as the cell cycle of budding yeast (Irons 2009). Dynamical modules are detectable in whole-genome data sets (Alexander et al. 2009), but have not yet been formally identified and characterised in developmental systems other than those mentioned above. Plenty of evidence suggests that they will be in the near future. Here we name a few likely candidates. The AC/DC circuit involved in patterning of the vertebrate neural tube exhibits all the criteria to qualify it as a dynamical module: within its region of influence, it is responsible for the main patterning function of the system it is embedded in (Balaskas et al. 2012, Panovska-Griffiths et al. 2013). Turing pattern generators compose another, very broad, class of candidate dynamical modules (Turing 1952, Meinhardt 1982, Meinhardt \& Gierer 2010, Kondo \& Miura 2010, Marcon \& Sharpe 2012, Green \& Sharpe 2015). Specifically, the core mechanism underlying proximo-distal and digit patterning in the vertebrate limb, from shark fins to tetrapod appendages, can be seen as a module that dynamically interacts with tissue geometry and morphogens within the growing limb bud (Raspopovic et al. 2014, Onimaru et al. 2016). Various systems driving cellular rhythms should be decomposable in terms of dynamical modularity (Goldbeter 1997, Novák \& Tyson 2008, Maroto \& Monk 2009). The logical analysis in Irons (2009) could be generalised and extended to continuous models of the cell cycle (see, for example, Chen et al. 2000, 2004; reviewed in Tyson \& Novák, 2015). Circadian oscillations provide another interesting case study (Goldbeter 1995, Asgari-Targhi \& Klerman 2018), for which Boolean models exist (Akman et al. 2012) that are amenable to analysis with the algorithm by Irons and Monk (2007). Dynamical modules have also been described in plants, particularly in patterning the root and leaf epidermis in Arabidopsis thaliana (Benítez et al. 2008, 2010, 2011). This diverse set of examples could be extended as one wishes, but it should be apparent that dynamical modules are omnipresent in cellular and developmental regulatory systems across the kingdoms of life.

Upon closer inspection, a hierarchy of dynamical modules can be discerned. Let us take the segmentation gene network of Drosophila as an example. This network in its entirety can be considered a module. It governs a particular aspect of the dynamics of pattern formation that is 
clearly discernible from other patterning processes. The segmentation gene system can be further subdivided into maternal co-ordinate, gap, pair-rule, and segment-polarity subsystems (Fig. 2A). Again, these subsystems affect separable dynamic aspects of body patterning (Nüsslein-Volhard \& Wieschaus 1980). They interact with other processes, such as those triggered by homeotic genes that specify the identity of body segments (e.g. Akam 1987, Ingham 1988). Our work on the gap gene system further divides the network down to the AC/DC subcircuit (Fig. 6D) (Verd et al. 2019). Irons and Monk (2007) partition the segmentpolarity network into even smaller subunits of intercellular feedback mechanisms (Fig. $5 \mathrm{H}$ ). Dynamical decomposition reveals that the dynamic repertoire of systems at higher levels in the hierarchy is dissociable into lower-level dynamical regimes.

This hierarchy of dynamical modules bears striking similarities to the hierarchy of morphogenetic fields from classical embryology (Gilbert et al. 1996). Especially for higher levels of the hierarchy, we can draw a close parallel between dynamical modules and the field concept. The notion of a morphogenetic field was first introduced by Theodor Boveri in 1910, and was further developed by biologists such as Gurwitsch, Weiss, Harrison, and Needham, who refined its definition and provided experimental support for field phenomena in embryology. In its original meaning, the morphogenetic field is not an incipient undifferentiated tissue, but rather a concept representing the interactions of localized developmental processes to generate a robust pattern (Raff 1996; see also Goodwin 1982, Goodwin et al. 1993, Webster \& Goodwin 1996). In this sense, it exhibits obvious similarities to the notion of a developmental module: morphogenetic fields have a cohesive internal regulatory structure and generate dissociable patterns (Needham 1933). They interact with other fields in a hierarchical and dynamic way: the primary embryonic field, for example, yields to more organ-specific fields over time, which are refined as embryogenesis proceeds (Raff 1996). In essence, therefore, a dynamical module is a formally defined representation of a morphogenetic field. And, as classical embryology already recognised, morphogenetic fields are the foundational unit of developmental process.

\section{Evolutionary implications}

Dynamical modularity has important implications for evolution. We have explained in the "Introduction" why modular traits require functional modularity in the genotype-phenotype map as a prerequisite for evolvability (Wagner \& Altenberg 1996). We have argued in line with Simon $(1962,1973)$ that near-decomposability (or dissociability, Needham 1933) is a fundamental property of complex adaptive systems. It enables modules to vary relatively independently, minimizing off-target pleiotropic effects, which accounts for the individuality of character traits (Wagner \& Altenberg 1996, Wagner \& Zhang 2011). This kind of functional modularity provides a causal-mechanistic explanation for character identity and homology (Wagner 2007, 2014). In the section "Structural modules," we have discussed how modules can be co-opted into new pathways during evolution, generating innovative change (Raff 1996, von Dassow \& Munro 1999, True \& Carroll 2002, Davidson \& Erwin 2006, Erwin \& Davidson 2009, Monteiro \& 
Podlaha 2009, Wagner 2011). How do dynamical modules fit into this picture?

First of all, quasi-independence of character traits implies functional modularity in the generative processes or dynamics that constitute the underlying genotype-phenotype map (Fig. 1) (e.g. Waddington 1957, Goodwin 1982, Wagner 1989, Alberch 1991, Amundson 2005, Jaeger et al. 2012, Jaeger and Monk 2014, Wagner 2014). Different types of generative dynamics must therefore be identifiable and separable in some sense. In engineered systems, dissociability is achieved through modular structure: the components of a laptop screen, for example, map to the trait "display," while the components of the keyboard map to trait "input." These traits can evolve independently by altering the respective structural components. Integration is achieved by simple input-output maps between the different components. Some aspects of living systems may have evolved to be like this. However, more generally, we expect tighter structural integration, making it difficult to separate modules at the level of network structure (Jiménez et al. 2017). Wimsatt (2007) calls this type of integration "interactional complexity," arguing that it is a natural outcome of selective evolution. Dynamical modules capture the sense in which the genotype-map map of such systems can still be dissociated, even if it is not structurally decomposable at all.

A key point is that a given structure (network) in genotype space corresponds not to a single dynamical behaviour, but to a repertoire of different dynamic regimes. This repertoire is captured by the set of attractors and their basins. Note that it is typical, not unusual, that a dynamical system has multiple attractors for a single set of parameters: which attractor is manifested depends on the initial and boundary conditions (the context) of the network. In case of the gap gene network, for example, the different attractors are selected by maternal inputs (Manu et al. 2009, Verd et al. 2017, 2018). Dynamical modules give a functional decomposition of the repertoire associated with a given structural network. They reveal the separable aspects of the dynamics of the system. Dissociability in the dynamics is not simply related to the decomposability of the structure. This is important in the context of evolution because we can now explore how different dynamical modules respond to changes in the genotype.

Jiménez et al. (2017), and our study of the gap gene system (Verd et al. 2019), illustrate the sometimes extensive structural overlap between dynamical modules (Fig. 4D,E, Fig. 6C). At first sight, we must assume that mutations affecting genes or regulatory interactions involved in two or more modules are bound to cause pleiotropic effects. This seems incompatible with limited pleiotropy and the functional dissociability of modules. How can we explain this? The answer lies in the fact that, despite their structural overlap, dynamical modules can show vastly different sensitivities to mutational changes (Verd et al. 2019). In the case of the gap gene network, for example, subcircuits AC/DC1 and AC/DC3 are structurally stable and thus robust. In contrast, AC/DC2 is in a state of criticality, close to a bifurcation boundary, and thus sensitive to even subtle changes in its regulatory interactions. Accordingly, we observe that the overall dynamics and relative arrangement of gap domains is strongly conserved, while the position of the boundary between stable switch-like and shifting oscillatory dynamics differs markedly between Drosophila and another dipteran species, the scuttle fly Megaselia abdita 
(Wotton et al., 2015, Crombach et al., 2016). This suggests that AC/DC1 is the critical subcircuit in Megaselia, while AC/DC2 is a structurally stable damped oscillator (Fig. 6C) (Verd et al. 2019). This switch in criticality requires surprisingly few and subtle changes in gap gene cross-regulatory interactions.

Such differential sensitivity to change is an important aspect of evolvability that structural or regulatory modules cannot explain, since it requires a causal-mechanistic understanding of the generative process that produces the pattern (Goodwin 1982, Jaeger et al. 2012, Jaeger \& Monk 2014). The surprising and counterintuitive insight is that, even though genes and interactions are shared between modules, their effects on the dynamical behaviour of each module are different. This provides an alternative explanation for limited pleiotropy. It corroborates that the structure of networks and regulatory sequences does not necessarily have to be modular to ensure dissociability of functional modules. In fact, structurally more modular (hierarchical) networks can be dynamically less modular, since network structure and the richness of a system's dynamical repertoire are only loosely associated. The prevalence of modular structures in the literature may therefore result from ascertainment bias, rather than a true reflection of the organization of regulatory systems, because, modular networks and regulatory structure are easier to detect and characterise than the dynamical modularity described here.

The definition of dynamical module in Irons and Monk (2007) requires that a module has a dynamical behaviour that is conserved in multiple different attractors. If the module corresponds to a trait, then evolution can shift the system as a whole to a new attractor, while preserving the trait in question. There is a set of attractors for the whole system, which all incorporate the same subsystem for the trait to be conserved. This subsystem can stay conserved, even if other aspects of the attractors change during evolution. In this way, the decomposition of the dynamical repertoire of a network into dynamical modules gives understanding of the evolutionary potential of the system as a whole. The geometrical arrangement of attractors and their basins in the phase space of the system determines the likelihood of phenotypic transitions (Alberch 1991; Jaeger and Monk 2014). Transitions between neighboring basins are facilitated by large borders between them, while transitions between attractor basins that do not border each other can only be indirect, through intermediate phenotypes. In most cases, phase space geometry will be complex, which means that systems with trivially dissociable genotype-phenotype maps are rare. The norm will be maps that have multiple discernable aspects to their dynamics that are "smeared" across overlapping regions of the network topology. To achieve decomposition in these cases, we need to use techniques that interrogate the dynamics in detail.

Finally, we have seen in the section on "Functional modules" that the identification of subsystems responsible for conferring character identity can provide a powerful conceptual foundation for a mechanistic theory of homology (Wagner 2007, 2014). We also highlighted why both functional-genetic decomposition and approaches based on structural modularity fall short of reliably identifying and delineating such subsystems. Dynamical modules provide a 
powerful alternative. The dynamic behaviour of a subnetwork is much more closely related to its function than its regulatory structure. Moreover, dynamical modules provide a causalmechanistic explanation of the generative process underlying the trait, which accounts for its particular variational properties (Altenberg, 1995; Wagner \& Alternberg 1996, Salazar-Ciudad 2006). Dynamical modules are morphogenetic fields. Therefore, morphogenetic fields, rather than ChIN networks or structural modules, provide the causal-mechanistic foundation for trait homology. This has always been the case, from classical embryology to the present day (Gilbert et al. 1996). It strongly resonates with the concept of process homology, introduced by Gilbert \& Bolker (2001). Homology of process strives to identify "the ways in which homologous processes are regulated, replicated and changed over time" with the aim "to better understand how changes in development generate changes in morphology" (ibid., p. 10). Process homology is required to make sense of modular functions. Dynamical modules are required to make sense of process homology. The two go hand in hand.

\section{Conclusions}

Dynamical modules provide a powerful approach to characterize the morphogenetic fields that form the causal-mechanistic basis for the functional modularity of the genotype-phenotype map and the homology of character traits. Dynamical modularity is much more closely related to function than structural or regulatory modules. We demonstrated the practical feasibility of identifying and characterising such modules in models of specific regulatory systems. We outlined promising candidates for the identification of additional dynamical modules. Finally, we discussed the evolutionary implications of our conceptual framework. The identification and characterisation of dynamical modules is an essential component of any theory aiming to mechanistically explain the origin and variability of character traits in terms of the generative processes that produce them (Goodwin 1982, Wagner \& Alternberg 1996, Wagner et al. 2000).

Characterisation of dynamic modules not only requires decomposition by genetic and molecular approaches, but also the reconstitution of their cohesive internal structure and their coherent integrated activity using dynamical modeling. As von Dassow \& Munro (1999, p. 309) stated, mechanism is "an explanatory mode in which we describe what are the parts, how they behave intrinsically, and how those parts are coupled to each other to produce the behavior of the whole." This last point is emphasised in the framework of dynamic mechanistic explanation (Bechtel \& Richardson 1993, Bechtel \& Abrahamsen 2005, 2010, Bechtel 2011) which focuses on the ability of mechanisms to account for patterns of activity and change over time. To understand these dynamic patterns we need models. "[M]odeling provides understanding beyond that which is available from identifying the parts, operations, and organization of the mechanism and mentally rehearsing its functioning" (Bechtel 2012, p. 244; see also von Dassow \& Meir 2004). This approach is an explicit elaboration and refinement of Simon's theoretical framework of near-decomposability (Bechtel \& Richardson 1993; see also Callebaut 2005). 
As a practical caveat, we point out that it will always be challenging and laborious to rigorously reverse-engineer dynamical modules, since this requires extensive empirical evidence tightly combined with dynamical models of the regulatory process under study. There are good practical reasons why structural approaches have dominated the field: structural modules are relatively easy to identify. It may well be that we have to confine dynamical decomposition to a limited sample of tractable model systems. We are convinced that even such a limited sample would yield interesting and generalisable insights into the causal-mechanistic principles underlying trait individuality, identity, and homology. These principles can be used for the mechanistic interpretation of results from correlational approaches to variational modules based on quantitative genetics (e.g. Wagner \& Zhang 2011, Nunes et al. 2013). Combined, the two complementary methodologies yield a powerful and general approach to the study of phenotypic evolution.

Another well-known pitfall is to confuse homology at the level of developmental process with homology of characters. The hierarchical organisation and robust behaviour of developmental modules implies that many different molecular mechanisms can lead to the same dynamical behaviour and, therefore, phenotypic outcome (Waddington 1957, Oster \& Alberch, 1982, Goodwin et al. 1993, Wagner 2011, Jaeger \& Monk 2014). This means that evolution at the genotypic and phenotypic level are at least partially dissociable (von Dassow \& Munro 1999, Bolker 2000, Gilbert \& Bolker 2001). An important consequence of dissociability is network or developmental system drift ${ }^{16}$ (Weiss \& Fullerton 2000, True \& Haag 2001, Wagner 2011, Pavlicev \& Wagner 2012). Regulatory mechanisms can evolve quite freely by rewiring network connections, as long as the phenotypic output of the process remains the same (see Wagner 2011, Jaeger \& Monk 2014). Process homology of dynamical modules must therefore be established at the level of the epigenotype as the underlying mechanisms can vary considerably even if the dynamics of the process are conserved (Fig. 1B). Even worse, entirely non-homologous generative processes can produce homologous character traits. A good example is insect segmentation: while many insects establish their segments in parallel by partitioning the embryo into territories as in Drosophila, this mode of segment determination is derived from an ancestral process that generates segments sequentially through growth and terminal addition (Sander 1976, Davis \& Patel 2002, Rosenberg et al. 2009, Lynch et al. 2012). The resulting segmented body plan is undoubtedly homologous at the morphological level, yet the underlying processes have diverged radically during evolution.

This provides both a challenge and an opportunity for the study of phenotypic evolution: "[r]ather than view dissociability as a problem for comparative biologists let us recognize it as an architectural feature of evolvable developmental systems, a feature whose origins and consequences deserve attention" (von Dassow \& Munro 1999). We mainly focused on the aspect of identifying and characterising developmental modules, providing the basis for a new research programme for developmental evolution. Our review of the existing literature on modularity indicates this programme is best pursued by a combination of empirical research,

16. Also called phenogenetic drift (Weiss \& Fullerton 2000, Weiss 2005). 
ensemble modelling approaches, and data-driven dynamical modelling. Empirical research is necessary for functional decomposition to identify the components of a dynamical modules. Decomposition is and remains an essential precondition for the mechanistic study of phenotypic evolution. Ensemble approaches then provide a map of regulatory possibilities, which helps us to frame our concepts and questions. The study by Jiménez et al. (2017), for example, pointed us to the fact that most multifunctional networks may not be structurally modular, and that structural modules are not required for functional modularity. These are two important but highly counterintuitive conclusions. Finally, reverse-engineering specific regulatory systems provides us with the link between functional evidence from decomposition and the possibilities provided by ensemble modelling (Jaeger \& Crombach, 2012, Green et al. 2015, Crombach \& Jaeger, this volume). Together, these complementary approaches will yield a completely new level of mechanistic understanding of the genotype-phenotype map, and its role in character trait evolution.

\section{References}

Ahnert SE, Fink T (2016) Form and function in gene regulatory networks: the structure of network motifs determines fundamental properties of their dynamical state space. $J$ Roy Soc Interface 13: 20160179

Akam M (1987) The molecular basis for metameric pattern in the Drosophila embryo. Development 101: $1-22$

Akman OE, Watterson S, Parton A, Binns N, Millar AJ, Ghazal P (2012). Digital clocks: simple Boolean models can quantitatively describe circadian systems. J Roy Soc Interface 9: 2365-2382.

Alberch P (1991) From genes to phenotype: dynamical systems and evolvability. Genetica 84: 5-11

Albert R, Othmer H (2003) The topology of the regulatory interactions predicts the expression pattern of the segment polarity genes in Drosophila. J Theor Biol 223: 1-18

Alexander RP, Kim PM, Emonet T, Gerstein MB (2009). Understanding modularity in molecular networks requires dynamics. Sci Signaling 2: pe44

Alon U (2006) An introduction to systems biology: design principles of biological circuits. Chapman and Hall/CRC, London

Alon U (2007) Network motifs: theory and experimental approaches. Nat Rev Genet 8: 450-461

Altenberg L (1995) Genome growth and the evolution of the genotype-phenotype map. In: Banzhaf W, Eeckman FH (eds) Evolution and biocomputation: computational models of evolution. Springer, Berlin

Amundson $\mathrm{R}$ (2005) The changing role of the embryo in evolutionary thought. Cambridge University Press, Cambridge

Asgari-Targhi A, Klerman EB (2018) Mathematical modeling of circadian rhythms. WIREs Syst Biol Med e1439 
Ashyraliyev M, Siggens K, Janssens H, Blom J, Akam M, Jaeger J (2009) Gene circuit analysis of the terminal gap gene huckebein. PLoS Comp Biol 5: e1000548

Babu MM, Luscombe NM, Aravind L, Gerstein M, Teichmann SA (2004) Structure and evolution of transcriptional regulatory networks. Curr Op Struct Biol 14: 283-291

Balaskas N, Ribeiro A, Panovska J, Dessaud E, Sasai N, Page KM, Briscoe J, Ribes V (2012) Gene regulatory logic for reading the Sonic Hedgehog signaling gradient in the vertebrate neural tube. Cell 148: $273-284$

Bar-Joseph Z, Gerber GK, Lee TI, Rinaldi NJ, Yoo JY, Robert F, Gordon DB, Fraenkel E, Jaakkola TS, Young RA, Gifford DK (2003) Computational discovery of gene modules and regulatory networks. Nat Biotech 21: 1337-1342

Bechtel W, Richardson RC (1993) Discovering complexity: decomposition and localization as strategies in scientific research. MIT Press, Cambridge MA

Bechtel W, Abrahamsen A (2005) Explanation: a mechanist alternative. Stud Hist Phil Biol \& Biomed Sci 36: $421-441$

Bechtel W, Abrahamsen A (2010) Dynamic mechanistic explanation: computational modeling of circadian rhythms as an exemplar for cognitive science. Stud Hist Phil Sci A 41: 321-333

Bechtel W (2011) Mechanism and biological explanation. Phil Sci 78: 533-557

Bechtel W (2012) Understanding endogenously active mechanisms: a scientific and philosophical challenge. Eur J Phil Sci 2: 233-248

Benítez M, Espinosa-Soto C, Padilla-Longoria P, Alvarez-Buylla ER (2008). Interlinked nonlinear subnetworks underlie the formation of robust cellular patterns in Arabidopsis epidermis: a dynamical spatial model. BMC Syst Biol 2: 98

Benítez M, Alvarez-Buylla ER (2010). Dynamic-module redundancy confers robustness to the gene regulatory network involved in hair patterning of Arabidopsis epidermis. BioSystems 102: 11-15

Benítez M, Monk NA, Alvarez-Buylla ER (2011). Epidermal patterning in Arabidopsis: models make a difference. J Exp Zool (Mol Dev Evol) 316: 241-253

Benson AR, Gleich DF, Leskovec J (2016) Higher-order organization of complex networks. Science 353: 163-166

Bolker JA (2000) Modularity in development and why it matters to evo-devo. Amer Zool 40: 770-776

Bolouri H (2014) Modeling genomic regulatory networks with big data. Trends Genet 30: 182-191

Bonner JT (1988) The evolution of complexity by means of natural selection. Princeton University Press, Princeton NJ

Brandon RN (1999) The units of selection revisited: the modules of selection. Biol Philos 14: 167-180

Briscoe J, Small S (2015) Morphogen rules: design principles of gradient-mediated embryo patterning. Development 142: 3996-4009

Burda Z, Krzywicki A, Martin OC, Zagorski M (2011). Motifs emerge from function in model gene regulatory networks. Proc Natl Acad Sci U.S.A. 108: 17263-17268 
Burns J (1970) The synthetic problem and the genotype-phenotype relation in cellular metabolism. In: Waddington $\mathrm{CH}$ (ed) Towards a Theoretical Biology, Vol. III. Edinburgh University Press, Edinburgh

Callebaut W (2005) The ubiquity of modularity. In: Callebaut W, Rasskin-Gutman D (eds) ModularityUnderstanding the development and evolution of natural complex systems. MIT Press, Cambridge, MA

Callebaut W, Rasskin-Gutman D (2005) Modularity-Understanding the development and evolution of natural complex systems. MIT Press, Cambridge, MA

Chaves M, Albert R, Sontag E (2005) Robustness and fragility of Boolean models for genetic regulatory networks. J Theor Biol 235: 431-449

Chen KC, Csikasz-Nagy A, Gyorffy B, Val J, Novak B, Tyson JJ (2000) Kinetic analysis of a molecular model of the budding yeast cell cycle. Mol Biol Cell 11: 369-391

Chen KC, Calzone L, Csikasz-Nagy A, Cross FR, Novak B, Tyson JJ (2004) Integrative analysis of cell cycle control in budding yeast. Mol Biol Cell 15: 3841-3862

Christensen WD, Bickhard MH (2002) The process dynamics of normative function. The Monist 85: 3-28

Collier J (1988) Supervenience and reduction in biological hierarchies. Can J Phil 14: 209-234

Collier J (2004) Self-organization, individuation and identity. Rev Intl Phil 14: 209-234

Cotterell J, Sharpe J (2010) An atlas of gene regulatory networks reveals multiple three-gene mechanisms for interpreting morphogen gradients. Mol Syst Biol 6: 425

Crombach A, Hogeweg P (2008) Evolution of evolvability in gene regulatory networks. PLoS Comp Biol 4: e1000112

Crombach A, Wotton KR, Cicin-Sain D, Ashyraliyev M, Jaeger J (2012) Efficient reverse-engineering of a developmental gene regulatory network. PLoS Comp Biol 8: e1002589

Crombach A, Wotton KR, Jiménez-Guri E, Jaeger J (2016) Gap gene regulatory dynamics evolve along a genotype network. Mol Biol Evol 33: 1293-1307

Cummins (1975) Functional analysis. J Phil 72: 741-765

Davidson EH, Rast JP, Oliveri P, Ransick A, Calestani C, Yuh C-H, Minokawa T, Amore G, Hinman V, Arenas-Mena C, Otim O, Brown CT, Livi CB, Lee PY, Revilla R, Rust AG, Pan ZJ, Schilstra MJ, Clarke PJC, Arnone MI, Rowen L, Cameron RA, McClay DR, Hood L, Bolouri H (2002) A genomic regulatory network for development. Science 295: 1669-1678

Davidson EH, Erwin DH (2006) Gene regulatory networks and the evolution of animal body plans. Science 311: 796-800.

Davidson EH (2010) Emerging properties of animal gene regulatory networks. Nature 468: 911-920

Davidson EH (2011) Evolutionary bioscience as regulatory systems biology. Dev Biol 357: 35-40

Davis GK, Patel NH (2002) Short, long, and beyond: molecular and embryological approaches to insect segmentation. Ann Rev Entomol 47: 669-699

Dawkins R (1989) The evolution of evolvability. In: Langton C (ed) Artificial life: the proceedings of an interdisciplinary workshop on the synthesis and simulation of living systems. Addison-Wesley, Redwood City CA 
DiFrisco J (2018) Biological processes: criteria of identity and persistence. In: Nicholson DJ, Dupré J (eds) Everything flows: towards a processual philosophy of biology. Oxford University Press, Oxford

DiFrisco J, Jaeger J (2019). Beyond networks: mechanism and process in Evo-Devo. Biol Philos 34: 54

DiFrisco J, Love AC, Wagner GP (2020). Character identity mechanisms: a conceptual model for comparative-mechanistic biology. Biol Philos 35: 44

Dobrin R, Beg QK, Barabási A-L, Oltvai ZN (2004) Aggregation of topological motifs in the Escherichia coli transcriptional regulatory network. BMC Bioinformatics 5: 10

Eisen MB, Spellman PT, Brown PO, Botstein D (1998) Cluster analysis and display of genome-wide expression patterns. Proc Natl Acad Sci U.S.A. 95: 14863-14868

Erwin DH, Davidson EH (2009) The evolution of hierarchical gene regulatory networks. Nat Rev Genet 10: $141-148$

Fortunato S (2010) Community detection in graphs. Phys Rep 486: 75-174

Gilbert SF, Opitz JM, Raff RA (1996) Resynthesizing evolutionary and developmental biology. Dev Biol 173: 357-372

Gilbert SF, Bolker JA (2001) Homologies of process and modular elements of embryonic construction. $J$ Exp Zool (Mol Dev Evol) 291: 1-12

Girvan M, Newman ME (2002) Community structure in social and biological networks. Proc Natl Acad Sci U.S.A. 99: 7821-7826

Green S, Fagan M, Jaeger J (2015) Explanatory integration challenges in evolutionary systems biology. Biol Theor 10: 18-35

Goldbeter A (1995) A model for circadian oscillations in the Drosophila period protein (PER). Proc $R$ Soc Lond B 261: 319-324

Goldbeter A (1997) Biochemical oscillations and cellular rhythms: the molecular bases of periodic and chaotic behaviour. Cambridge University Press, Cambridge

Goodwin BC (1982) Development and evolution. J Theor Biol 97: 43-55

Goodwin BC, Kauffman SA, Murray JD (1993) Is morphogenesis an intrinsically robust process? J Theor Biol 163: 135-144

Gorochowski TE, Grierson CS, di Bernardo M (2018). Organization of feed-forward loop motifs reveals architectural principles in natural and engineered networks. Sci Adv 4: eaap9751

Gould SJ, Lewontin RC (1979). The spandrels of San Marco and the Panglossian paradigm: a critique of the adaptationist programme. Proc Roy Soc London B 205: 581-598

Green JBA, Sharpe J (2015) Positional information and reaction-diffusion: two big ideas in developmental biology combine. Development 142: 1203-1211

Gursky VV, Panok L, Myasnikova EM, Manu, Samsonova MG, Reinitz J, Samsonov AM (2011) Mechanisms of gap gene expression canalization in the Drosophila blastoderm. BMC Syst Biol 5: 118

Hartwell LH, Hopfield JJ, Leibler S, Murray AW (1999) From molecular to modular cell biology. Nature 402 (Suppl.): C47-C52 
Hernández-Hernández V, Niklas KJ, Newman SA, Benítez M (2012). Dynamical patterning modules in plant development and evolution. Intl J Dev Biol 56: 661-674.

Hull DL (1980) Individuality and selection. Ann Rev Ecol Syst 11: 311-332

Ingham PW (1988) The molecular genetics of embryonic pattern formation in Drosophila. Nature 335: 2534

Ingram PJ, Stumpf MP, Stark J (2006) Network motifs: structure does not determine function. BMC Genomics 7: 108

Irons DJ, Monk NAM (2007) Identifying dynamical modules from genetic regulatory systems: applications to the segment polarity network. BMC Bioinformatics 8: 413

Irons DJ (2009) Logical analysis of the budding yeast cell cycle. J Theor Biol 257: 543-559

Isalan M, Morrison M (2009) This title is false. Nature 458: 969

Isalan M (2009) Gene networks and liar paradoxes. BioEssays 31: 1110-1115

Ishihara S, Shibata T (2008). Mutual interaction in network motifs robustly sharpens gene expression in developmental processes. J Theor Biol 252: 131-144

Jaeger J, Surkova S, Blagov M, Janssens H, Kosman D, Kozlov KN, Manu, Myasnikova E, VanarioAlonso CE, Samsonova M, Sharp DH, Reinitz J (2004a). Dynamic control of positional information in the early Drosophila embryo. Nature 430: 368-371

Jaeger J, Blagov M, Kosman D, Kozlov KN, Manu, Myasnikova E, Surkova S, Vanario-Alonso CE, Samsonova M, Sharp DH, Reinitz J (2004b) Dynamical analysis of regulatory interactions in the gap gene system of Drosophila melanogaster. Genetics 167: 1721-1737

Jaeger J, Irons D, Monk N (2008) Regulative feedback in pattern formation: towards a general relativistic theory of positional information. Development 135: 3175-3183

Jaeger J (2011) The gap gene network. Cell Mol Life Sci 68: 243-274

Jaeger J \& Crombach A (2012) Life's attractors: understanding developmental systems through reverse engineering and in silico evolution. In: Soyer O (ed.) Evolutionary systems biology. Springer, Berlin

Jaeger J, Irons D, Monk N (2012) The inheritance of process: a dynamical systems approach. J Exp Zool (Mol Dev Evol) 318B: 591-612

Jaeger J, Monk N (2014) Bioattractors: dynamical systems theory and the evolution of regulatory processes. J Physiol 592: 2267-2281

Jaeger J, Monk N (2015) Everything flows. EMBO Rep 16: 1064-1067

Jaeger J (2018) Shift happens: the developmental and evolutionary dynamics of the gap gene system. Curr Op Syst Biol 11: 65-73

Jiménez A, Cotterell J, Munteanu A, Sharpe J (2017) A spectrum of modularity in multi- functional gene circuits. Mol Syst Biol 13: 925

Kashtan N, Itzkovitz S, Milo R, Alon U (2004). Topological generalizations of network motifs. Phys Rev $E$ 70: 031909 
Kauffman SA (1969) Metabolic stability and epigenesis in randomly constructed genetic nets. $J$ Theor Biol 22: 437-467

Kauffman SA (1974) The large scale structure and dynamics of gene control circuits. J Theor Biol 44: $167-190$

Kauffman SA (1987) Developmental logic and its evolution. BioEssays 6: 82-87

Kauffman SA (1993) The origins of order: self organization and selection in evolution. Oxford University Press, Oxford

Kim M-S, Kim J-R, Cho K-H (2011) Dynamic network rewiring determines temporal regulatory functions in Drosophila melanogaster development processes. BioEssays 32: 505-513

Kondo S, Miura T (2010) Reaction-diffusion model as a framework for understanding biological pattern formation. Science 329: 1616-1620

Levine M, Davidson EH (2005) Gene regulatory networks for development. Proc Natl Acad Sci U.S.A. 102: 4936-4942

Lewontin RC (1970) The units of selection. Ann Rev Ecol Syst 1: 1-18

Lewontin RC (1974) The genetic basis of evolutionary change. Columbia University Press, New York

Lynch JA, El-Sherif E, Brown SJ (2012) Comparisons of the embryonic development of Drosophila, Nasonia, and Tribolium. Wiley Interdiscipl Rev: Dev Biol 1: 16-39

Ma W, Lai L, Ouyang Q, Tang C (2006) Robustness and modular design of the Drosophila segment polarity network. Mol Syst Biol 2: 70

Ma W, Trusina A, El-Samad H, Lim WA, Tang C (2009) Defining network topologies that can achieve biochemical adaptation. Cell 138: 760-773

Mangan S, Alon U (2003) Structure and function of the feed-forward loop network motif. Proc Natl Acad Sci U.S.A. 100: 11980-11985

Manu, Surkova S, Spirov AV, Gursky V, Janssens H, Kim AR, Radulescu O, Vanario-Alonso C, Sharp DH, Samsonova M, Reinitz J (2009) Canalization of gene expression and domain shifts in the Drosophila blastoderm by dynamical attractors. PLoS Comp Biol 5: e1000303

Marcon L, Sharpe J (2012) Turing patterns in development: what about the horse part? Curr Op Genet Dev 22: 578-584

Maroto M, Monk NAM (eds) (2009) Cellular oscillatory mechanisms. Landes Bioscience/Springer, New York

Mazurie A, Bottani S, Vergassola M (2005) An evolutionary and functional assessment of regulatory network motifs. Genome Biol 6: R35

Meinhardt H (1982) Models of biological pattern formation. Academic Press, London

Meinhardt H, Gierer A (2010) Pattern formation by local self-activation and lateral inhibition. BioEssays 22: $753-760$

Melo D, Porto A, Cheverud JM, Marroig G (2016). Modularity: genes, development, and evolution. Annu Rev Ecol Evol Syst 47: 463-486 
Milo R, Shen-Orr S, Itzkovitz S, Kashtan N, Chklovskii D, Alon U (2002). Network motifs: simple building blocks of complex networks. Science 298: 824-827

Milo R, Itzkovitz S, Kashtan N, Levitt R, Shen-Orr S, Ayzenshtat I, Sheffer M, Alon U (2004) Superfamilies of evolved and designed networks. Science 303: 1538-1542

Mireles V, Conrad TO (2018). Reusable building blocks in biological systems. J Roy Soc Interface 15: 20180595

Monteiro A, Podlaha O (2009) Wings, horns, and butterfly eyespots: how do complex traits evolve? PLoS Biol 7: e1000037

Moss L (2001) Deconstructing the gene and reconstructing molecular developmental systems. In: Oyama S, Griffiths PE, Gray RD (eds) Cycles of contingency: developmental systems and evolution. MIT Press, Cambridge MA

Mossio M, Saborido C, Moreno A (2009). An organizational account of biological functions. Brit J Phil Sci 60: 813-841

Needham J (1933). On the dissociability of the fundamental processes in ontogenesis. Biol Rev 8: 180223

Newman MEJ (2006) Modularity and community structure in networks. Proc Natl Acad Sci U.S.A. 103: 8577-8582

Newman MEJ, Barabási A-L, Watts DJ (2006). The structure and dynamics of networks. Princeton University Press, Princeton NJ

Newman SA, Bhat R (2008). Dynamical patterning modules: physico-genetic determinants of morphological development and evolution. Phys Biol 5: 015008

Newman SA, Bhat R (2009). Dynamical patterning modules: a "pattern language" for development and evolution of multicellular form. Intl J Dev Biol 53: 693-705

Nicholson DJ, Dupré J (2018) Everything flows: towards a processual philosophy of biology. Oxford University Press, Oxford

Niehrs C (2004) Synexpression Groups: Genetic Modules and Embryonic Development. In: Schlosser G, Wagner GP (eds) Modularity in Development and Evolution. University of Chicago Press, Chicago

Novák B, Tyson J (2008) Design principles of biochemical oscillators. Nat Rev Mol Cell Biol 9: 981-991

Nunes MDS, Arif S, Schlötterer C, McGregor AP (2013) A perspective on micro-evo-devo: progress and potential. Genetics 195: 625-634

Nüsslein-Volhard C, Wieschaus E (1980) Mutations affecting segment number and polarity in Drosophila. Nature 287: 795-801

Oliveri P, Davidson EH (2004). Gene regulatory network controlling embryonic specification in the sea urchin. Curr Op Genet Dev 14: 351-360

Onimaru K, Marcon L, Musy M, Tanaka M, Sharpe J (2016) The fin-to-limb transition as the reorganization of a Turing pattern. Nat Comms 7: 11582

Oster G, Alberch P (1982) Evolution and bifurcation of developmental programs. Evolution 36: 444-459 
Page KM, Perez-Carrasco R (2018) Degradation rate uniformity determines success of oscillations in repressive feedback regulatory networks. J Roy Soc Interface 15: 20180157

Palla G, Derényi I, Farkas I, Vicsek T (2005) Uncovering the overlapping community structure of complex networks in nature and society. Nature 435: 814-818

Panovska-Griffiths J, Page KM, Briscoe J (2013). A gene regulatory motif that generates oscillatory or multiway switch outputs. J Roy Soc Interface 15: 20180157

Papatsenko D (2009). Stripe formation in the early fly embryo: principles, models, and networks. BioEssays 31: 1172-1180.

Pavlicev M, Wagner GP (2012) A model of developmental evolution: selection, pleiotropy and compensation. Trends Ecol Evol 27: 316-322

Payne JL, Wanger A (2015). Function does not follow form in gene regulatory circuits. Sci Rep 5: 13015

Perez-Carrasco R, Barnes CP, Schaerli Y, Isalan M, Briscoe J, Page KM (2018). Combining a toggle switch and a repressilator within the AC-DC circuit generates distinct dynamical behaviors. Cell Systems 5: $521-530$

Peter IS, Davidson EH (2011) Evolution of gene regulatory networks controlling body plan development. Cell 144: 970-985

Peter IS, Faure E, Davidson EH (2012). Predictive computation of genomic logic processing functions in embryonic development. Proc Natl Acad Sci U.S.A. 109: 16434-16442

Peter IS, Davidson EH (2017) Assessing regulatory information in developmental gene regulatory networks. Proc Natl Acad Sci U.S.A. 114: 5862-5869

Pigliucci M (2008) Is evolvability evolvable? Nat Rev Genet 9: 75-82

Pigliucci M (2010) Genotype-phenotype mapping and the end of the 'genes as a blueprint' metaphor. Phil Trans $R$ Soc $B$ 365: 557-566

Radicchi F, Castellano C, Cecconi F, Loreto V, Parisi D (2004) Defining and identifying communities in networks. Proc Natl Acad Sci U.S.A. 101: 2658-2663

Raff RA (1996) The shape of life: genes, development, and the evolution of animal form. Chicago University Press, Chicago

Raspopovic J, Marcon L, Russo L, Sharpe J (2014) Digit patterning is controlled by a Bmp- Sox9-Wnt Turing network modulated by morphogen gradients. Science 345: 566-570

Riedl R (1975) Die Ordnung des Lebendigen: Systembedingungen der Evolution. Parey, Hamburg/Berlin (English translation: Riedl R (auth.), Jeffries RPS (transl.) (1978) Order in living systems: a systems analysis of evolution. Wiley, New York)

Rosenberg MI, Lynch JA, Desplan C (2009) Heads and tails: evolution of antero-posterior patterning in insects. Biochim Biophys Acta 1789: 333-342

Rosenfeld N, Alon U (2003) Response delays and the structure of transcription networks. J Mol Biol 329: 645-65 
Salazar-Ciudad I, Garcia-Fernández J, Solé RV (2000) Gene networks capable of pattern formation: from induction to reaction-diffusion. $J$ Theor Biol 205: 587-603

Salazar-Ciudad I, Newman SA, Solé RV (2001a) Phenotypic and dynamical transitions in model genetic networks I. Emergence of patterns and genotype-phenotype relationships. Evol Dev 3: 84-94

Salazar-Ciudad I, Solé RV, Newman SA (2001b) Phenotypic and dynamical transitions in model genetic networks II. Application to the evolution of segmentation mechanisms. Evol Dev 3: 95-103

Salazar-Ciudad I (2006) Developmental constraints vs. variational properties: how pattern formation can help to understand evolution and development. J Exp Zool (Mol Dev Evol) 306B: 107-125

Sander K (1976) Specification of the basic body pattern in insect embryogenesis. Adv Insect Physiol 12: 124-238

Schlosser G, Wagner GP (2004). Modularity in Development and Evolution. University of Chicago Press, Chicago

Segal E, Shapira M, Regev A, Pe'er D, Botstein D, Koller D, Friedman N (2003). Module networks: identifying regulatory modules and their condition-specific regulators from gene expression data. Nat Genet 34: 166-176

Shellman ER, Burant CF, Schnell S (2013). Network motifs provide signatures that characterize metabolism. Mol BioSyst 9: 352

Shen-Orr SS, Milo R, Mangan S, Alon U (2002). Network motifs in the transcriptional regulation network of Escherichia coli. Nat Genet 31: 64-68

Siebert H (2009) Dynamical and structural modularity of discrete regulatory networks. In: Back RJ, Petre I, de Vink E (eds) Computational Models for Cell Processes (CompMod 2009) EPTCS 6:109-124

Siebert $\mathrm{H}$ (2011) Analysis of discrete bioregulatory networks using symbolic steady states. Bull Math Biol 73:873-898

Siegal ML, Promislow DEL, Bergman A (2007) Functional and evolutionary inference in gene networks: does topology matter? Genetica 129: 83-103

Simon HA (1962) The architecture of complexity. Proc Amer Phil Soc 106: 467-482

Simon HA (1973) The organization of complex systems. In: Pattee HH (ed) Hierarchy theory. George Braziller, New York

Solé RV, Valverde S (2006) Are network motifs the spandrels of cellular complexity? Trends Ecol Evol 21: 419-422

Strogatz SH (2014) Nonlinear dynamics and chaos-with applications to physics, biology, chemistry, and engineering. Westview Press, Boulder CA

Stuart JM, Segal E, Koller D, Kim SK (2003) A gene-coexpression network for global discovery of conserved genetic modules. Science 302: 249-255

Tavazoie S, Hughes JD, Campbell MJ, Cho RJ, Church GM (1999) Systematic determination of genetic network architecture. Nat Genet 22: 281-285 
Teichmann SA, Babu MM (2002) Conservation of gene co-regulation in prokaryotes and eukaryotes. Trends Biotechnol 20: 407-410

True JR, Haag ES (2001) Developmental system drift and flexibility in evolutionary trajectories. Evol Dev 3: $109-119$

True JR, Carroll SB (2002) Gene co-option in physiological and morphological evolution. Ann Rev Cell Dev Biol 18: 53-80

Turing AM (1952) The chemical basis of morphogenesis. Phil Trans $R$ Soc Lond B 237: 37-72

Tyson JJ, Novák B (2015) Models in biology: lessons from modeling regulation of the eukaryotic cell cycle. BMC Biology 13: 46

Verd B, Crombach A, Jaeger J (2017) Dynamic maternal gradients control timing and shift-rates for Drosophila gap gene expression. PLoS Comp Biol 13: e1005285

Verd B, Clark E, Wotton KR, Janssens H, Jiménez-Guri E, Crombach A, Jaeger J (2018) A damped oscillator imposes temporal order on posterior gap gene expression in Drosophila. PLoS Biol 16: e2003174

Verd B, Monk NAM, Jaeger J (2019) Modularity, criticality, and evolvability of a developmental gene regulatory network. eLIFE 8: e42832

von Dassow G, Munro E (1999). Modularity in animal development and evolution: elements of a conceptual framework for EvoDevo. J Exp Zool (Mol Dev Evol) 285: 307-325

von Dassow G, Meir E (2004). Exploring modularity with dynamical models of gene networks. In: Schlosser G, Wagner GP (eds) Modularity in development and evolution. University of Chicago Press, Chicago

Waddington $\mathrm{CH}$ (1942) The epigenotype. Endeavour 1: 18-20 (reprinted in 2012: Int J Epidemiol 41: 1013)

Waddington CH (1953) Epigenetics and evolution. In: Symposia of the society for experimental biology VII: evolution. Cambridge University Press, Cambridge

Waddington $\mathrm{CH}$ (1957) The strategy of the genes: a discussion of some aspects of theoretical biology. Allen \& Unwin, London

Wagner A (2011). The origins of evolutionary innovations: a theory of transformative change in living systems. Oxford University Press, Oxford

Wagner GP (1989) The biological homology concept. Ann Rev Ecol Syst 20: 51-69

Wagner GP (1996) Homologues, natural kinds and the evolution of modularity. Amer Zool 36: 36-43

Wagner GP, Altenberg L (1996) Complex adaptations and the evolution of evolvability. Evolution 50: 967-976

Wagner GP, Chiu C-H, Laubichler M (2000) Developmental evolution as a mechanistic science: the inference from developmental mechanisms to evolutionary processes. Amer Zool 40: 819-831

Wagner GP (2007) The developmental genetics of homology. Nat Rev Genet 8: 473-479

Wagner GP, Pavlicev M, Cheverud JM (2007) The road to modularity. Nat Rev Genet 8: 921-931 
Wagner GP, Zhang J (2011). The pleiotropic structure of the genotype-phenotype map: the evolvability of complex organisms. Nat Rev Genet 12: 204-213

Wagner GP (2014) Homology, genes, and evolutionary innovation. Princeton University Press, Princeton

Wall ME, Dunlop MJ, Hlavacek S (2005) Multiple functions of a feed-forward-loop gene circuit. J Mol Biol 349: $501-514$

Webster G, Goodwin BC (1996). Form and transformation: generative and relational principles in biology. Cambridge University Press, Cambridge

Weiss KM, Fullterton SM (2000) Phenogenetic drift and the evolution of genotype-phenotype relationships. Theor Pop Biol 57: 187-195

Weiss KM (2005) The phenogenetic logic of life. Nat Rev Genet 6: 36-45

Wimsatt WC (2007) Re-engineering philosophy for limited beings: piecewise approximations to reality. Harvard University Press, Cambridge MA

Winther RG (2001) Varieties of modules: kinds, levels, origins, and behaviors. J Exp Zool (Mol Dev Evol) 291: $116-129$

Wolpert $L$ (1968) The French Flag problem: a contribution to the discussion on pattern development and regulation. In: Waddington $\mathrm{CH}$ (ed) Towards a Theoretical Biology, Vol. I. Edinburgh University Press, Edinburgh

Wolpert $L$ (1969) Positional information and the spatial pattern of cellular differentiation. $J$ Theor Biol 25 : $1-47$

Wotton KR, Jiménez-Guri, E, Crombach A, Janssens H, Alcaine-Colet A, Lemke S, Schmidt-Ott U, Jaeger $J$ (2015). Quantitative system drift compensates for altered maternal inputs to the gap gene network of the scuttle fly Megaselia abdita. eLIFE 4: e04785

Wright L (1973). Functions. Phil Rev 82: 139-168

Zinzen RP, Papatsenko D (2007). Enhancer responses to similarly distributed antagonistic gradients in development. PloS Comp Biol 3: e84 